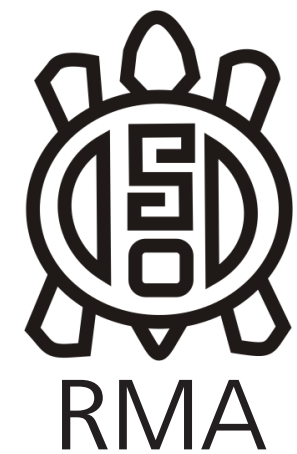

Arqueología

\title{
Cambios ambientales y estrategias de aprovisionamiento de rocas durante el periodo 12000-4000 años AP en la puna de Jujuy, Argentina
}

\author{
Environmental changes and supply rocks strategies of during the \\ period 12 000-4000 years AP in Jujuy puna, Argentina
}

Brenda Oxman* y Rodolphe Hoguin**

Universidad de Buenos Aires, faculta de Filosofía y Letras, Instituto de Arqueología, Argentina. CONICET. E mail: *brendaoxman@gmail.com **roddh2002@yahoo.fr

\begin{abstract}
Resumen
El presente trabajo tiene como objetivo explorar la relación entre los cambios ambientales producidos en la Puna Seca Argentina durante el periodo 12 000- 4000 años AP y su incidencia en la variabilidad observada en las estrategias adaptativas desarrolladas por los grupos humanos en la tecnología lítica. Las líneas de evidencia utilizadas son los análisis polínicos para el estudio del paleoambeinte y el aprovisionamiento de materias primas líticas como indicador de los rangos de movilidad de los grupos humanos. Los resultados polínicos obtenidos muestran que el Holoceno temprano habría sido más húmedo y con una menor distancia entre los parches de vegetación; mientras que durante el Holoceno medio se detectan variaciones ambientales y tecnológicasy el periodo se diferencia en dos momentos, que se ven reflejadas en una mayor especialización de los sitios en relación a las diferentes actividades tecnológicas, acompañado un proceso de aridez en aumento. Otro de los factores importantes es la variación ambiental que se observa en la segunda mitad del Holoceno medio, que pudo haber incidido en la toma de decisión de los grupos humanos frente a este factor de riesgo.
\end{abstract}

Palabras claves: Polen; paleoambiente; Puna; tecnología lítica; cazadores recolectores.

\begin{abstract}
The present paper aims to explore the relationship between the environmental changes produced in the Puna Seca Argentina during the period 12 000-4000 years BP and its incidence in the variability observed in the adaptive strategies developed by human groups in lithic technology. The lines of evidence used for the study of the paleoenvironment were mainly the pollen analyzes, and for the mobility it was used the technological analysis for the approach of supply of the different types of rocks. The results show that the Early Holocene was more stable and humid environment, with a smaller distance between patches of vegetation and those groups of hunter-gatherers developed a direct supply of certain raw materials and the rock-carving sequence was fully developed in the same residential base; while during the middle Holocene an environmental and technological variations are detected and the period were divided into two different moments, which is reflected in a higher specialization of the sites in relation to the different technological activities, accompanied by a growing aridity process. Likewise, an increase in the environmental variations in the second half of the middle Holocene is detected, which may have influenced the decision-making of human groups in relation to this risk factor.
\end{abstract}

Keywords: pollen; paleoenvironment; Puna; lithic technology; hunter gatherer.

Diversos estudios han demostrado que los cambios ambientales producen modificaciones en la estructura de recursos que afectan a las estrategias de subsistencias de los grupos humanos, generando a veces problemas que los grupos humanos deben resolver (Dincauze 2000). La tecnología lítica es una de las herramientas con las que cuentan los seres humanos para hacer frente a estas situaciones cambiantes (Fitzhugh 2001, entre otros). La movilidad se ve afectada por estos cambios, particularmente en el tiempo y en la recurrencia de las ocupaciones en los sitios, lo que requiere una adaptación de las estrategias de aprovisionamiento de materias primas (Kent 1992).

En la Puna Argentina el estudio de las variaciones ambientales y su posible incidencia en poblaciones humanas es de suma importancia, ya que esta área es definida como un desierto de altura y presenta ciertas limitaciones en cuanto a la disponibilidad de agua y la capacidad de sustento de biomasa primaria y secundaria. 
Actualmente se puede observar como pequeñas variaciones en el clima impactan fuertemente en este tipo de ambientes. La baja predictibilidad de las variaciones climáticas es un factor de riesgo para los seres humanos (Halstead y O'Shea 1989, Minc y Smith 1989, Bousman han ido cambiando a través de los años, por lo que su estudio es de fundamental relevancia para comprender la particular evolución del paisaje y los grupos humanos en el área.

El objetivo de este trabajo consiste en exponer los resultados de los estudios ambientales y arqueológicos, desarrollados a partir de la utilización de dos principales líneas de evidencias, en la Puna Seca argentina durante el periodo comprendido entre 12000-4000 años AP: la palinología como indicador de la composición de la vegetación, en estrecha relación con las variaciones producidas en las precipitaciones y la temperatura.

\section{La Puna: descripción del área y potenciales recursos para las poblaciones humanas}

El área de estudio corresponde a la Puna Seca Argentina, situada entre los $22^{\circ}$ y $24^{\circ} \mathrm{S}$ y entre los 3000 y 4500 msnm, definida como un bioma de desierto de altura. Se caracteriza por la alta radiación solar debido a su gran altitud, y gran amplitud térmica diaria, marcada estacionalidad de las precipitaciones, y una baja presión atmosférica. Las precipitaciones en el noroeste de Argentina están reguladas por el llamado monzón de América del Sur (Garreaud et al. 2009), produciéndose el $80 \%$ de las precipitaciones anuales durante los meses de diciembre y marzo (ca. 200 mm / año en la región de Susques) (Vuille y Keimig 2004). Estas características producen que la vegetación de la Puna se distribuya 1993). El ambiente no es estático y sus características

en un gradiente altitudinal, pudiéndose discriminar dos principales composiciones florísticas. Por una lado, se encuentra la vegetación de tolar (estepa arbustiva), conformada mayormente por especies de la familia Asteraceae; y por el otro, la de pajonal (estepa herbácea), representada mayormente por la familia Poaceae. Así también, se hallan las vegas (humedales) cuya distribución es azonal (Cabrera 1976), y están representadas mayormente por la familia Cyperaceae, Juncaceae y Poaceae. En la Puna este tipo de parches de vegetación representan zonas de concentración de recursos, debido a que cuenta con disponibilidad de agua y alta productividad primaria (Figura 1).

Los animales más importantes para la subsistencia humana en la Puna incluyen varios mamíferos (por ejemplo, la vicuña Vicugna vicugna y el guanaco Lama guanicoe), roedores (por ejemplo, vizcachas y chinchillas Lagidium viscacia y Chinchilla brevicaudata), y un cérvido (Taruca, Hippocamelus antisensis) (Yacobaccio 1994).

Dentro de las materias primas líticas potencialmente explotables para las poblaciones humanas, se encuentran la obsidiana, andesita, cuarcita y otras rocas silíceas. Las obsidianas son de particular importancia por sus cualidades para la talla de los artefactos y han sido transportadas desde largas distancias para su utilización. Para la región de estudio hay tres localidades que presentan variantes geoquímicas conspicuas: Zapaleri/Laguna Blanca, Caldera Vilama, Alto Tocomar, Quirón y Ona. Estos vidrios volcánicos se presentan bajo forma de nódulos pequeños, aunque pudiendo alcanzar $20 \mathrm{~cm}$ en algunas fuentes como la de Zapaleri (Yacobaccio et al. 2004). Estas fuentes primarias están concentradas en el paisaje y no aparecen dispersas en fuentes secundarias, salvo en Alto Tocomar. La cuarcita está disponible inmediatamente a los alrededores de todos los

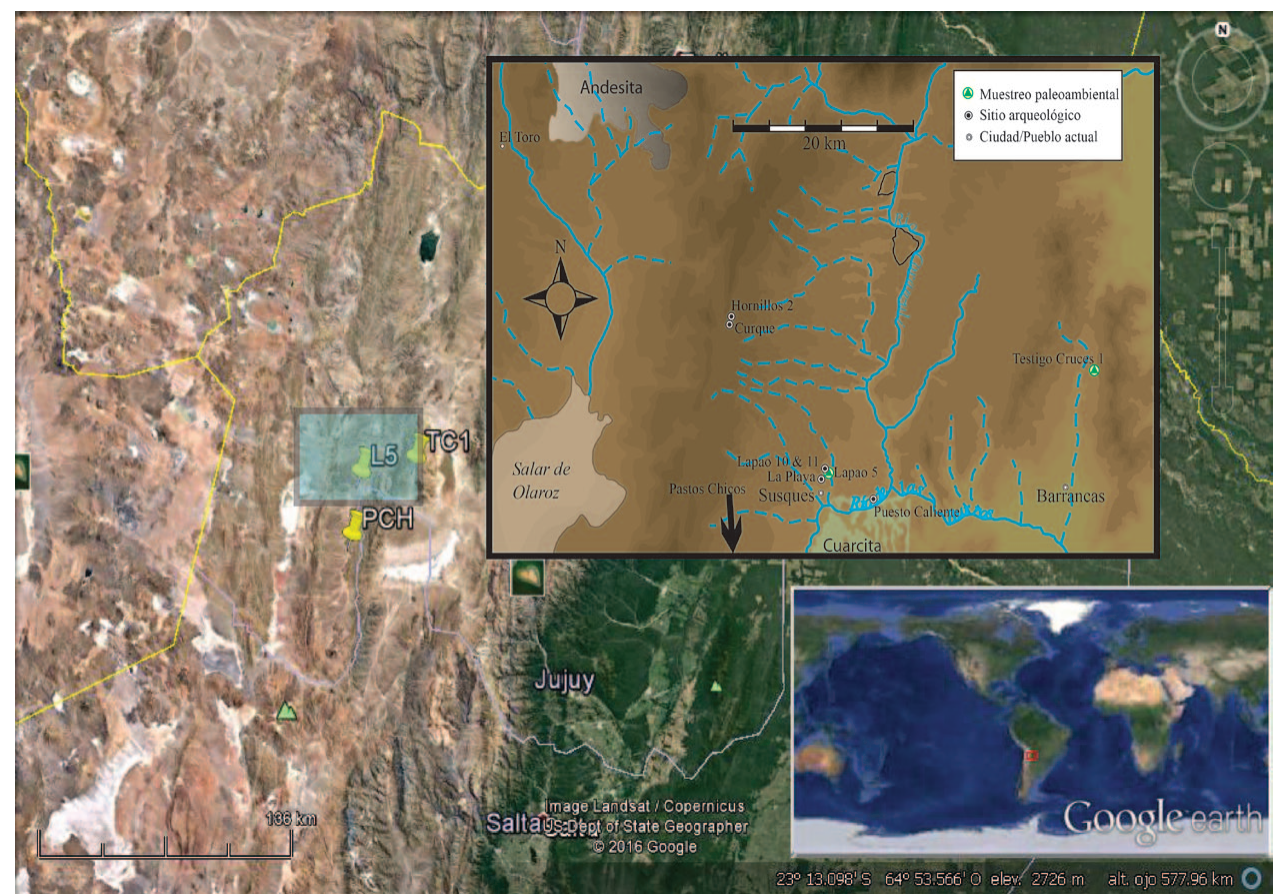
sitios analizados porque aflora en las sierras del Taire (carta geológica de Nullo, Solá com. pers.). Si bien los afloramientos primarios están presentes y son abundantes en el área de estudio, están acotados en el paisaje.

Figura 1. Mapa del área de estudio. Ubicación de las secuencias sedimentarias estudiadas y sitios arqueológicos seleccionados para el estudio de tecnología lítica

Figure 1. Map of the study area. Location of sedimentary sequences studied and archaeological sites selected for the study of lithic technology 
Aun siendo locales $(<10 \mathrm{~km})$, no suelen estar presentes en las inmediaciones de los sitios presentados. El tamaño de los bloques no restringe ningún tipo de extracción, pudiendo alcanzar hasta ca. $60 \mathrm{~cm}$ de longitud en algunos afloramientos primarios. Los afloramientos secundarios son más dispersos en el paisaje, pero proveen alta disponibilidad de bloques de tamaño más reducido ( 12-15 cm). En cuanto a la andesita, se trata de una roca volcánica para la cual solo se conoce una fuente principal a $\sim 40 \mathrm{~km}$ al Oeste de Hornillos 2, constituida por una extensa cantera-taller en la localidad de El Toro (Figura 1). Allí se presenta en una alta diversidad de bloques de distintos tamaños, algunos muy grandes pueden medir más de $1 \mathrm{~m}$ de altura (Yacobaccio y Morales com. pers.). El origen de estas andesitas podría ser los centros volcánicos máficos, los más cercanos de Hornillos 2 y de los sitios de la quebrada de Lapao siendo Campo Negro, Cerros del Toro y el cerro Negro de Olaroz (Maro y Caffe 2012). Varios filones de otras rocas más restringidos y menos frecuentes se pueden encontrar localmente. Se trata de varios tipos de rocas silíceas: riolita, cuarcita de grano fino, calcedonia, ftanita, ópalo, entre otros. Se puede también mencionar nódulos de ópalo presentes en facies marginales del salar de Olaroz, que se encuentran en tamaños pequeños (mayormente entre 5 y $10 \mathrm{~cm}$ ) y presentan superficies muy irregulares (Bustillo y Alonso 1989).

\section{Antecedentes en la Puna Argentina}

Antecedentes paleoambientales de la Puna Argentina Los estudios paleoambientales para el periodo de finales del Pleistoceno se limitan a los realizados en Barro Negro (Fernández et al. 1991) y Sierra de Santa Victoria (Schäbitz 2000). Los análisis polínicos e isotópicos (oxígeno y carbón) en Barro Negro describen un ambiente más húmedo que el actual entre 12500 - 10200 años $A P$, representado por el descenso de la estepa herbácea (en relación a su ubicación actual) por debajo de los 4100 m snm (Fernández et al. 1991) y los valores de isótopos que señalan condiciones más frías. También Schäbitz (2000) en Sierra de Santa Victoria muestra condiciones de más húmedas entre 11000 y 8500 años AP. Para el periodo del Holoceno Temprano en El Aguilar, Quebrada de Humahuaca, y también en la Puna de Salta se detectan condiciones más frías y humedad entre 10000 - 8000/7500 años AP (Markgraf 1985, Schäbitz et al. 2001). Sin embargo, en Barro Negro el retroceso de la estepa herbácea, como consecuencia de condiciones más cálidas, comienza ya hacia ca. 10200 años AP. Para el Holoceno medio, los resultados de Markgraf (1985) en El Aguilar evidencian un momento más seco entre 7500 - 4000 años AP. Asimismo, las conclusiones de Schäbitz et al. (2001) muestran condiciones de mayor aridez entre 8500 - 2000 años AP. También Lupo (1998), registra en Yavi un momento de aridez entre 8000 - 6000 años AP. En contraposición, en cotas altitudinales más altas, los estudios de la relación Carbón/Nitrógeno y diatomeas en Alto Tocomar (4300 msnm), han registrado condiciones de mayor humedad entre 8250 - 5700 años AP, con un máximo entre 6400 - 5700 años AP (Morales 2011). Para comienzos del Holoceno tardío, en la Cuenca del río Yavi los análisis sedimentológico, palinológico y arqueológico (Kulemeyer y Lupo 1994), muestran una vega estable entre 4500 - 4000 años AP y un aumento de sedimentación desde 4000 años AP. También, en cotas más altas como señalan los estudios en Chorcan, se detecta la presencia de una vega en un marco regional árido producto de una reducción de las precipitaciones entre ca. 5000- 2800 años AP (Schittek 2014) (ver tabla 1).

\section{Estudios paleoambientales en las localidades de Pastos} Chicos y Lapao

Los estudios paleoambientales en las localidades de Pastos Chicos y Lapao consisten en el análisis de diatomeas, sedimentos, geomorfología y polen (Morales 2011, Oxman 2015, Tchilinguirian et al 2012, Oxman y Yacobaccio 2012, Pirola 2014).

En Lapao 5, los antecedentes señalan tres zonaciones. La primera entre 9000 - 8850 años AP, dominada por las especies de diatomeas de forma de vida litoral, que indican la presencia de una vega moderadamente vegetada. Entre 8800 - 8100 años AP, se detecta un momento de aumento de las especies litorales y máxima humedad entre 8800 - 8600 años AP. Por último, entre 8100 - 7317 años AP, dominan las bentonitas, lo que marca la instalación de las condiciones de aridez (Morales 2011, Tchilinguirian et al. 2014). En el caso de Pastos Chicos, se llevaron a cabo análisis sedimentológicos (Tchilinguirian et al. 2012), diatomeas (Morales 2011) y materia orgánica (Pirola 2014). Los análisis distinguen dos momentos paleoambientales. El primero, un ambiente litoral, como una planicie de inundación, con desarrollo de turba y régimen de humedad permanente entre 9300 7400 años AP; y el segundo, a partir de 7400 años AP, con desarrollo de un humedal más seco y salobre, y pulsos de humedad entre 7050 - 6900 años AP. A estos se suman, los valores de materia orgánica ca. 7900 años AP, que muestran valores más bajos y altos valores de carbonatos entre 7900 - 7500 años AP (Morales 2011, Pirola 2014).

Antecedentes arqueológicos: movilidad y patrones de asentamiento

Las investigaciones arqueológicas sobre la movilidad y los patrones de asentamiento de cazadores-recolectores en la Puna de los Andes Centro-Sur se desarrollaron de manera significativa durante las últimas décadas. Los estudios paleoambientales y cronológicos de sitios arqueológicos permitieron entender mejor las primeras ocupaciones del final del Pleistoceno y del inicio del Holoceno temprano. En particular se planteó que la colonización de la Puna se realizó desde los espacios más bajos en base a la cronología de los sitios y en relación a los requisitos de una ocupación efectiva para la adaptación humana (Aldenderfer 1998, Muscio 1998-1999, Osorio et al. 2017, Yacobaccio y Morales 2013, entre otros). Los 
estudios de aprovisionamiento de materias primas en el Noroeste argentino, en sentido amplio (incluyendo recursos faunísticos y vegetales), contribuyeron mucho a la discusión, indagando sobre la complementariedad entre ambientes. Por ejemplo en la Puna Salada, se destaca la presencia de materiales vegetales procedentes de las yungas e inclusive del chaco, así como cuentas del Pacífico, aunque en menor cantidad (Mondini et al. 2013, Martínez 2012, Pintar 2014, Pintar y Rodríguez 2015). La presencia de bienes exóticos procedentes de otros pisos ecológicos de la vertiente oriental es también notable en los sitios de la Quebrada de Humahuaca (Aguerre et al. 1975, Fernández Distel 1974, Hernández Llosas 2000). Las investigaciones apuntan por lo general a una movilidad alta de pequeños grupos humanos durante el Holoceno temprano (Aschero 1994, Loyola et al. 2017, López 2008, Yacobaccio y Morales 2013).

Sin embargo, Susques (nuestra área de estudio) parece haber funcionado de manera distinta. Si bien no se niega la probabilidad alta de algún tipo de acceso a recursos críticos alóctonos como las cañas para los astiles (corroborados indirectamente por la presencia de puntas de proyectil con elementos de prensión axial tal como pedúnculos y escotadura entre otros), los pigmentos y las obsidianas halladas en las capas del Holoceno temprano de Hornillos 2 evidencian rangos de acción limitados a la Puna (Yacobaccio et al. 2008). Los estudios de aprovisionamiento de obsidianas en la Puna de Salta y de Jujuy parecen mostrar una movilidad siguiendo un eje norte-sur (Mercuri y Restifo 2014, Yacobaccio et al. 2017). Asimismo para sitios de otras áreas de los Andes Centro-Sur donde ya desde el Holoceno temprano, existiría una ocupación local y permanente de las tierras

\begin{tabular}{|c|c|c|c|c|}
\hline \multicolumn{5}{|c|}{ ESTUDIOS PALEOAMBIENTALES EN LA PUNA ARGENTINA } \\
\hline \multicolumn{5}{|c|}{ PLEISTOCENO-HOLOCENO } \\
\hline $\begin{array}{l}\text { Cronología AP } \\
\text { (en miles de } \\
\text { años) }\end{array}$ & Localidad & $\begin{array}{l}\text { Línea de } \\
\text { evidencia }\end{array}$ & $\begin{array}{c}\text { Interpretació } \\
\mathrm{n} \text { ambiental }\end{array}$ & Referencia \\
\hline $9,5-8,5$ & Cordillera oriental (Jujuy) & Geomorfología & Húmedo y frío & $\begin{array}{l}\text { Zipprich et al. } \\
2000\end{array}$ \\
\hline 13 & $\begin{array}{c}\text { Sierra de Santa Victoria } \\
\text { (Jujuy) }\end{array}$ & Geomorfología & Húmedo y frío & $\begin{array}{c}\text { Zech et al. (2009, } \\
\text { 2011) }\end{array}$ \\
\hline $12,5-10,2$ & Barro Negro (Jujuy) & Polen e isótopos & Húmedo y frío & $\begin{array}{c}\text { Fernández et al. } \\
\text { (1991) }\end{array}$ \\
\hline \multicolumn{5}{|c|}{ HOLOCENO TEMPRANO } \\
\hline $10-7,5$ & El Aguilar (Jujuy) & Polen & Húmedo y frío & Markgraf (1985) \\
\hline $11-8,5$ & Puna de Jujuy y Salta & Polen & Húmedo & Shäbitz et al. 2001 \\
\hline \multicolumn{5}{|c|}{ HOLOCENO MEDIO } \\
\hline $7,5-4$ & El Aguilar & Polen & Seco y cálido & Markgraf (1985) \\
\hline $8,5-2$ & $\begin{array}{c}\text { La Palca, Río Yavi, Laguna } \\
\text { Grande, Tres Lagunas y Abra } \\
\text { de La Cruz (Jujuy) }\end{array}$ & $\begin{array}{c}\text { Polen y } \\
\text { sedimentología }\end{array}$ & Seco & $\begin{array}{c}\text { Shäbitz et al. } \\
\text { (2001) }\end{array}$ \\
\hline 8- 6 & Yavi (Jujuy) & Polen & Húmedo & Lupo (1998) \\
\hline $8,9-8,2$ & \multirow[t]{2}{*}{ Río Chico (Jujuy) } & \multirow[t]{2}{*}{ Sedimentología } & Húmedo & \multirow[t]{2}{*}{$\begin{array}{c}\text { Alcalde y } \\
\text { Kulemeyer (1999) }\end{array}$} \\
\hline 6,4 & & & Seco & \\
\hline $8,2-5,7$ & Alto Tocomar (Jujuy) & $\mathrm{C} / \mathrm{N}$ y diatomeas & Húmedo & Morales (2011) \\
\hline $5-2,8$ & Chorcán, Salta & Polen y carbón & Seco & Schittek (2014) \\
\hline
\end{tabular}

altas, dejando los espacios costeros (Aldenderfer 1998, Osorio et al. 2017).

Otro tema que trataron los estudios de aprovisionamiento, en particular de estudios de fuentes de obsidiana, es sobre el conocimiento sobre el ambiente local, siendo menor en una primera etapa de la ocupación de un espacio menos efectiva como por ejemplo en los sitios de Antofagasta de la Sierra (Pintar et al. 2016). En otras áreas tales como la Quebrada Maní del Norte de Chile parece ocurrir un fenómeno contrario, donde la selección de los recursos combustibles reflejan un fehaciente conocimiento y uso del ambiente local por las ocupaciones tempranas (Joly et al. 2017).

Por lo general, hay un consenso que durante el Holoceno medio hay una disminución de la movilidad residencial en un ambiente fragmentado de mayor aridez generalizada con una restricción de los parches de recursos, explotados por una movilidad de tipo logístico (Aschero 1994, Aschero y Martínez 2001, López 2008, Pintar et al. 2016, Pintar y Rodríguez 2015, Yacobaccio y Morales 2005). También, el aumento general de la señal arqueológica parece resultar de un aumento del tamaño de los grupos (Muscio y López 2017). Varios hiatos e interrupciones de la señal arqueológica podrían documentar procesos de dispersión y/o abandono intermitente de ciertas localidades (Muscio y López 2017, Pintar 2014, Yacobaccio 2013). El acceso a otros pisos ecológicos no fue por lo tanto abandonado. En Antofagasta de la Sierra se planteó la hipótesis del "Puna-Plus", según la cual los grupos siguen una movilidad estacional logística para el acceso a áreas extra-puneñas (Mondini et al. 2013), tal como lo confirmaría la evidencia arqueológica de sitios en valles mesotermales (Martínez et al. 2013). En este sentido, en la Puna de Salta, se detectó también la presencia de recursos alóctonos, tales como una cuenta de valva marítima procedente de la costa Pacífica (López 2008, López y Orsi 2017). Se indagó en particular sobre un cambio desde una movilidad de tipo forager por "salto" durante el Holoceno temprano, hacia una movilidad de tipo logístico de "punto a punto" durante el Holoceno medio (Pintar y Rodríguez 2015). La disminución de la movilidad residencial es concomitante con el inicio de una

Tabla 1. Resumen de los antecedentes paleoambientales de la Puna Argentina para el periodo de estudio

Table 1. Summary of the paleoenvironmental history of the Argentine Puna for the study period 
caza más selectiva y de la domesticación de los camélidos (López y Orsi 2017, Mondini et al. 2013, Yacobaccio 2013, Yacobaccio y Morales 2005, entre otros). En cuanto a los análisis de procedencia de obsidiana, muestran una diversificación de las fuentes, interpretada como el resultado de un mayor conocimiento del ambiente por parte de las poblaciones (Pintar et al. 2016). En Susques, se observa nuevamente un fenómeno contrario con el análisis de las obsidianas, con una diversificación más acotada de las fuentes, siendo casi exclusivamente norteñas (Zapaleri y Caldera Vilama) durante el Holoceno medio, abandonando las fuentes sureñas utilizadas durante el Holoceno temprano, mostrando así una disminución de la movilidad (Yacobaccio et al. 2017).

Nuevamente, tampoco se niega algún tipo de contacto con otros pisos ecológicos para el abastecimiento de recursos críticos. En efecto, se compararon diseños de puntas de proyectil muy similares entre valles y Puna en Salta, planteando algún grado de transmisión entre grupos distantes (Restifo y Carbonelli 2016).

Tal como lo podemos observar, en la Puna de los Andes Centro-Sur y particularmente en la Puna argentina, las distintas líneas de evidencia en las distintas regiones muestran una diversidad de procesos y los registros arqueológicos exponen ciertas singularidades. Asimismo, los factores que controlan la selección de materias primas son variados en cada región y un mismo resultado puede ser interpretado de manera distinta. En efecto, es necesario tomar en cuenta la diversidad de factores a tomar en cuenta en interpretar los estudios relacionados con el aprovisionamiento de materias primas, no siempre ligados a la movilidad. Por ejemplo, en la Puna de Salta se planteó que la elección preferencial de la andesita local era principalmente por los requerimientos de ciertas tecnologías orientadas a la producción de hojas, y no solamente por la reducción de la movilidad (Restifo 2017).

En base a la variabilidad observada tanto en los registros paleoambientales como en los antecedentes arqueológicos, este trabajo propone avanzar en el estudio de la particular historia de las variaciones ambientales en la Puna Seca Argentina y las estrategias de aprovisionamiento de rocas que los grupos humanos desarrollaron para adaptarse y sobrevivir en uno de los actuales desiertos más áridos del mundo.

Líneas de evidencias: análisis polínicos y aprovisionamiento de materias primas.

Puesto que la vegetación responde en línea directa a las condiciones de humedad y temperatura, las variaciones en la composición de la vegetación permiten inferir variaciones climáticas como puede ser modificaciones en los patrones de las precipitaciones y la temperatura. Con este objetivo se ha aplicado un índice de humedad, que se calcula a partir de las variaciones en los porcentajes de las dos principales e indicadoras formaciones vegetacionales de la Puna (estepa herbácea y estepa arbustiva), de todos los registros polínicos trabajados por el equipo en el área para el periodo de investigación de este trabajo: Lapao 5 y Pastos Chicos 1-2 (Oxman y Yacobaccio 2014, Tchilinguirian et al. 2012; 2014, Tchilinguirian et al. 2014 a y b, Morales 2011, Oxman et al. 2013) situados en el departamento de Susques y el Testigo Cruces 1 situado en la localidad de Barrancas en el departamento de Cochinoca (Morales et al. 2016).

La información polínica generada será utilizada para delinear el escenario ambiental a escala regional en el que se desarrollaron determinados tipos de estrategias tecnológicas como medio para resolver la obtención y procesamiento de los recursos alimenticios para la subsistencia de los grupos humanos. Particularmente, se indagará el estudio del aprovisionamiento de las rocas utilizadas para la confección de instrumentos líticos en los sitios del área de Susques datados entre 100004000 años AP (Hoguin 2015). En efecto, la descripción de los afloramientos, su localización, la presentación morfológica de los bloques, su aptitud para la talla, su disponibilidad, abundancia y distribución son variables que permiten determinar restricciones y posibilidades e informarnos sobre la elección y las estrategias de aprovisionamiento en materias primas (Inizan et al. 1995). Se exponen dichas características geomorfológicas, luego se presentan las proporciones de las materias primas representadas en las muestras de los sitios y capas seleccionados para esta investigación. Esta base de información nos permitirá avanzar sobre el estudio de las diferentes actividades de talla realizadas en los sitios (desbaste, façonnage, retoque, entre otros). También, el análisis de los porcentajes de instrumentos y de lascas de desbaste (lascas de descortezamiento incluidas) permitirá investigar las actividades de transporte y las actividades realizadas en los sitios (Kent 1992).

\section{Materiales y Métodos}

\section{Análisis polínicos}

Descripción de las secuencias sedimentarias seleccionadas Las secuencias sedimentarias seleccionadas para ser

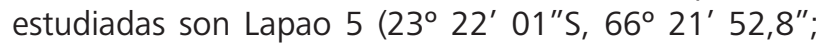
$3650 \mathrm{msnm}$ ) cuyo modelo cronológico abarca el periodo 9680-7021 años AP, Pastos chicos (23 40' 29'S, 66 25' 32"; 3781 msnm) que comprende el periodo ca. 4200 - 9256 años AP y el testigo Cruces 1 ( ${ }^{\circ} 18^{\prime}$ S; W 66 5 '12.9 "; 3682 msnm) representa el periodo 11650- 3936 años AP (Tabla 2).

En esta oportunidad se describe brevemente el muestreo y características de la secuencia sedimentaria del Testigo Cruces 1, ya que esta no ha sido presentada trabajos anteriores. El testigo comprende 2,66 $\mathrm{m}$ de profundidad y se seleccionaron 29 muestras, tomadas cada $8 \mathrm{~cm}$. La secuencia sedimentaria está compuesta por dos facies sedimentarias. La primera se extiende desde la superficie 
hasta los $2 \mathrm{~m}$. Son arcillas masivas y laminadas con materia orgánica, de colores grises y se observan macrorestos vegetales. La segunda facie se sitúa en los $20 \mathrm{~cm}$ del testigo y son arenas finas y limos. El contacto es de tipo erosivo porque hay un salto de edad de 11650 a 6300 años AP.

Para estimar la cronología de TC1 se efectuaron cuatro dataciones por el método de C14, aplicando la técnica de AMS (Tabla 2). También se calibraron las fechas con el programa Calib. Rev 7.0.2 (Stuiver y Reimer 1993) (ShCal13 14C; Hogget al. 2013). Con estos datos se confeccionó un modelo de edad profundidad. La tasa de sedimentación es de 13,8 años/cm entre 40 y 183 cm, y

Tabla 2. Fechados de las secuencias sedimentarias estudiadas

Table 2. Dates of the sedimentary sequences studied

\begin{tabular}{|c|c|c|c|c|c|c|}
\hline Cod. Lab. & $\begin{array}{l}\text { Cronología } \\
\text { C14 AP }\end{array}$ & Error & Profund. & Cal.2Sigmas & $\begin{array}{l}\text { Punto } \\
\text { medio }\end{array}$ & Referencia \\
\hline \multicolumn{7}{|c|}{$\mathrm{PCH}$} \\
\hline AA79835 & 4203 & 58 & 31 & $4528-4839$ & 4683 & $\begin{array}{c}\text { Morales 2011, Tchilinguirian et } \\
\text { al. } 2014\end{array}$ \\
\hline AA94570 & 6935 & 69 & 91 & $7595-7862$ & 7728 & $\begin{array}{c}\text { Morales 2011, Tchilinguirian et } \\
\text { al. } 2014\end{array}$ \\
\hline LP1836 & 7900 & 100 & 250 & $8454-8989$ & 8721 & $\begin{array}{c}\text { Morales } 2011 \text {, Tchilinguirian et } \\
\text { al. } 2014\end{array}$ \\
\hline LP1337 & 8900 & 130 & 331 & $9562-9573$ & 9567 & $\begin{array}{c}\text { Morales 2011, Tchilinguirian et } \\
\text { al. } 2014\end{array}$ \\
\hline LP981 & 7770 & 80 & 185 & $\begin{array}{l}\text { LAPAO } 5 \\
8374-8704\end{array}$ & 8539 & $\begin{array}{c}\text { Yacobaccio y Morales } 2005 \\
\text { Morales } 2011, \text { Tchilinguirian et } \\
\text { al. } 2012\end{array}$ \\
\hline LP1518 & 8380 & 100 & 270 & $9034-9525$ & 9279 & $\begin{array}{c}\text { Yacobaccio y Morales 2005, } \\
\text { Morales 2011, Tchilinguirian et } \\
\text { al. } 2012\end{array}$ \\
\hline LP1509 & 8560 & 90 & 310 & 9296-9696 & 9496 & $\begin{array}{c}\text { Yacobaccio y Morales 2005, } \\
\text { Morales 2011, Tchilinguirian et } \\
\text { al. } 2012\end{array}$ \\
\hline LP1534 & 9280 & 100 & 370 & 10226-10669 & 10447 & $\begin{array}{c}\text { Yacobaccio y Morales 2005, } \\
\text { Morales 2011, Tchilinguirian et } \\
\text { al. } 2012\end{array}$ \\
\hline \multicolumn{7}{|c|}{ TC1 } \\
\hline AA103187 & 11650 & 62 & 265 & 13303-13564 & 13433 & Morales et al. 2016 \\
\hline AA105235 & 6374 & 75 & 239 & $7025-7424$ & 7224 & Morales et al. 2016 \\
\hline AA103191 & 5919 & 42 & 183 & 6557-6797 & 6677 & Morales et al. 2016 \\
\hline AA103192 & 4865 & 37 & 107 & $5470-5562$ & 5516 & Morales et al. 2016 \\
\hline
\end{tabular}

Tabla 3. Muestreo total de artefactos por materias primas y por locus

Table 3. Total sample of artifacts by raw materials and locus

\begin{tabular}{|c|c|c|c|c|c|c|c|c|c|}
\hline \multirow{2}{*}{$\begin{array}{l}\text { Sitio } \\
\text { Capa/ } \\
\text { Rocas }\end{array}$} & \multicolumn{4}{|c|}{ Hornillos 2} & \multirow[t]{2}{*}{ La Playa } & \multirow{2}{*}{\multicolumn{2}{|c|}{$\begin{array}{c}\text { Lapao } 11 \text { Curque } \\
\text { Superficie }\end{array}$}} & \multirow[t]{2}{*}{ Lapao 10} & \multirow[t]{2}{*}{ Total } \\
\hline & Capas 6 & Capa 4 & Capa 3 & Capa 2 & & & & & \\
\hline Cuarcita & 1560 & 824 & 222 & 798 & 633 & 179 & 137 & 240 & 4593 \\
\hline Andesita & 1038 & 758 & 153 & 479 & 145 & 41 & 124 & 32 & 2770 \\
\hline Obsidiana & 619 & 502 & 390 & 480 & 24 & 14 & 91 & 6 & 2126 \\
\hline Sílices & 585 & 436 & 222 & 564 & 32 & 15 & 34 & 4 & 1037 \\
\hline Total & 3802 & 2520 & 987 & 2321 & 834 & 249 & 386 & 282 & 11381 \\
\hline
\end{tabular}

Tabla 4. Fechados radiocarbónicos (no calibrados) del sitio Hornillos 2 (tomado de Yacobaccio et al. 2013)

Table 4. Radiocarbon dates (non-calibrated) from Hornillos 2 site (taken from Yacobaccio et al. 2013)

\begin{tabular}{clcc}
\hline Capa & Fechas AP (no cal.) & Código Laboratorio (Método) & Materiales fechados \\
\hline 2 & $6130 \pm 70$ & Beta-111392 (LSC) & Carbón \\
& $6340 \pm 110$ & UGA-7829 (LSC) & Carbón \\
3 & $7430 \pm 80$ & UGA-7830 (LSC) & Carbón \\
& $7760 \pm 160$ & UGA-8722 (LSC) & Carbón \\
4 & $8280 \pm 100$ & LP-757 (LSC) & Carbón \\
6 & $9150 \pm 50$ & UGA-8723 (AMS) & Carbón \\
& $9590 \pm 50$ & UGA-8724 (AMS) & Madera \\
$6 d$ & $9710 \pm 270$ & UGA-13550 (LSC) & Carbón \\
\hline
\end{tabular}

de 8,1 años/cm entre 183 y $246 \mathrm{~cm}$. El contacto erosivo detectado entre 11650 y 6374 años AP, es un hiato del que no podemos fehacientemente calcular la tasa de sedimentación. Posiblemente la presencia de arenas esté indicando una tasa de depositación más elevada que el tramo superior, donde dominan las arcillas.

\section{Trabajo de laboratorio y análisis de datos}

Los análisis polínicos siguieron el método estándar para polen del Cuaternario (Faegri and Iversen, 1989). La etapa de laboratorio consistió en la observación microscópica de las muestras bajo microscopio biológico Zeiss-Axiolab, y contando como mínimo 200 granos por muestra. La identificación de los tipos polínicos se basó en la bibliografía disponible para el área de estudio (Heusser, 1971; Markgraf y D'Antoni 1978) y la palinoteca del Laboratorio de Palinología de la Facultad de Ciencias Agrarias de la UNJu/Conicet. Los análisis estadísticos se limitaron a la aplicación de un índice de humedad que se calcula a partir del logaritmo de la relación entre los dos principales tipos de vegetación dominante: la estepa herbácea y arbustiva (H/A en adelante). Esta ecuación ha sido utilizada por múltiples autores en el área andina como un índice de humedad (Liu et al. 2005).

Aprovisionamiento de materias primas Se analizó el material lítico compatible con rocas consideradas aptas para la talla: cuarcitas, andesita, obsidiana y rocas silíceas. Las unidades de muestreo consideradas en este trabajo son los locii, y fueron agrupadas de la siguiente manera: las distintas capas del Holoceno temprano de Hornillos 2 (6, 6a, 6b, $6 c$ y $6 d$ ) que fueron consideradas en bloque (capa 6) dado que los fechados no permitieron una distinción cronológica. La capa 5 se trata de un relleno de canal sin material orgánico y estéril arqueológicamente, por lo que no ha sido fechada y tampoco se realizó el análisis del material lítico. Las otras capas (2, 3 y 4$)$ fueron tomadas cada una como un locus particular. Finalmente, los sitios de superficie: La Playa, Lapao 10 y 11, Curque, fueron 
también tomados cada uno como un locus. La muestra consistió en 11381 artefactos que se clasificaron por materia prima y locii (Tabla 3), considerando su cronología (Tabla 4).

Para estimar una cronología tentativa de los locii de superficie, se utilizaron los fechados de Hornillos 2 y estudios previos sobre la identificación de artefactos diagnósticos y análisis de correspondencia (Hoguin 2015). De esta manera, el conjunto de capas 6, 6a, b, c y d de Hornillos 2 (capas 6) fue tomado como único locus del Holoceno temprano. Las capas 3 y 4 de Hornillos 2 y la Playa fueron clasificados en el Holoceno medio I (85006200 años AP) según el orden siguiente: capa 4, La Playa y capa 3. La capa 2 de Hornillos 2, Lapao 10, Lapao11 y Curque, fueron clasificados en el Holoceno medio II (6200-4000 años AP) según el siguiente orden: capa 2, Lapao 11, Curque y Lapao 10.

El análisis de los materiales líticos también incluye el estudio de las restricciones de las rocas aptas para la talla (fuente primaria o secundaria), así como la localización, distribución en el paisaje y abundancia de los afloramientos. En particular para los bloques se tuvo en cuenta las dimensiones, morfología y calidad para la talla (según el tipo de percusión). A su vez, los materiales líticos serán clasificados en las distintas etapas de las cadenas operativas, realizando una distinción entre las etapas relacionadas a la formatización de núcleos y producción de formas base (desbaste), y a la formatización y reactivación de instrumentos (Inizan et al. 1995, Kent 1992). Se clasificaron entonces los artefactos según las categorías siguientes: lascas de descortezamiento de núcleos (etapas iniciales), lascas de formatización de núcleo y formas base (lascas de desbaste), lascas de formatización de instrumento (lascas de confección) e instrumentos. Por otra parte, se calcularon también los porcentajes relativos de las etapas de formatización de núcleo (desbaste) y de confección de instrumentos en el marco de análisis de cadenas operativas (Boëda 2013). Así, fueron realizados los porcentajes de las lascas de descortezamiento, de los productos de desbaste (formas base y lascas de formatización de núcleo), de los instrumentos (que son confeccionados sobre formas base), sin incluir las lascas de confección de instrumentos y los indeterminados. El porcentaje de las lascas de confección de instrumentos (mucho más numerosas) fue calculado separadamente sobre el total de los desechos (incluyendo las lascas de formatización de núcleos y los desechos indeterminados). Luego, la reactivación fue calculada sobre el total de las lascas de confección. Estas distintas clases artefactuales corresponden a etapas clave de las cadenas operativas líticas en el orden en que suceden. De esta manera, los porcentajes relativos pueden aportar información relacionada la movilidad de los grupos humanos (e.g. anticipación, tipo de actividades realizadas, traslado de determinados artefactos, etc.) (Inizan et al. 1995, Kent 1992). Asimismo, si una materia prima está poco representada en una categoría u otra (por su acceso, disponibilidad, distancia o por el volumen restrictivo de sus bloques) en relación a las otras, esta clasificación permitirá entender mejor los cambios en las estrategias de aprovisionamiento.

\section{Resultados}

\section{Análisis polínicos}

Los cambios en la abundancia relativa de herbáceas y arbustivas, las dos taxa dominantes en los registros de polen de la Puna Argentina, pueden ser representada a partir del cálculo de la relación logarítmica de estas dos familias. Basado en la ecología moderna y la distribución de estos dos taxones vegetales, la relación polínica $\mathrm{H}$ / A resulta ser un proxy de la humedad ambiental. Las gramíneas (Poaceae) suelen tener raíces poco profundas y tienden a proliferar en condiciones más húmedas, mientras que las plantas xerófitas de Asteraceae son arbustos profundamente arraigados que proliferan en

$$
\text { Lapao5 Log H/A }
$$

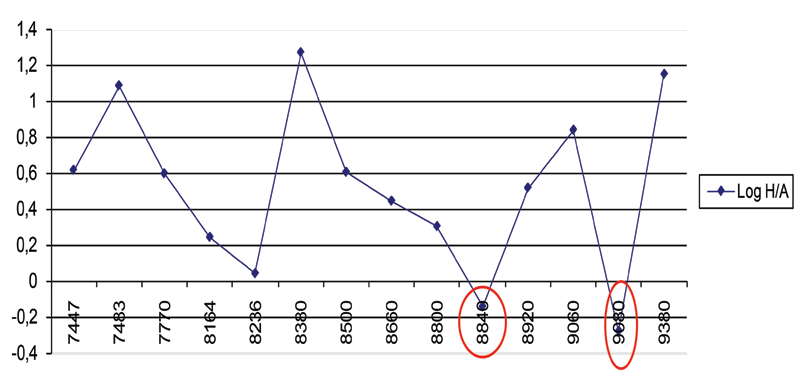

PCHLog H/A

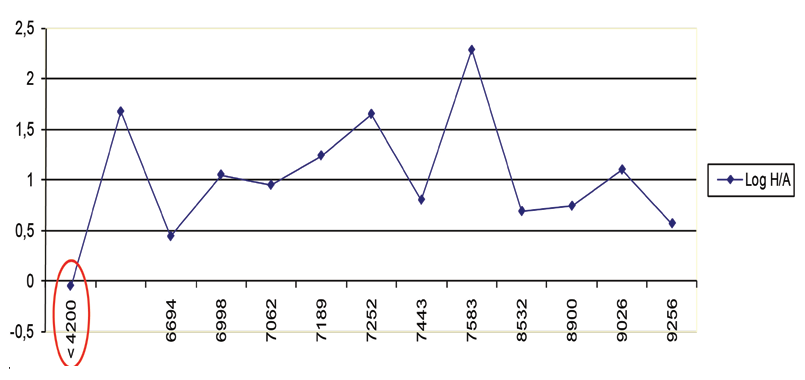

TC1 $\log H / A$

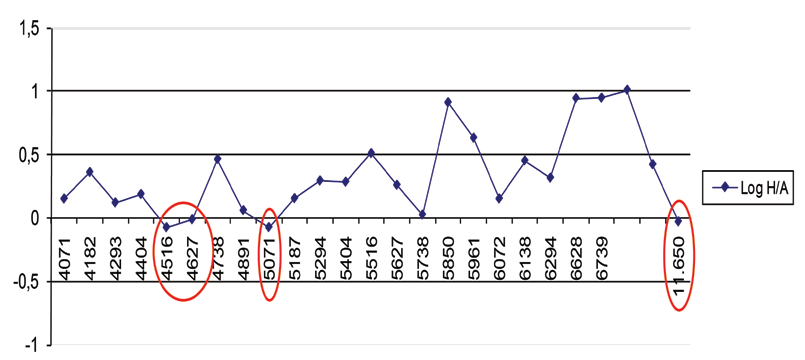

Figura 2. Gráfico del logaritmo H/A de las secuencias sedimentarias de Lapao 5, Pastos Chicos y del testigo TC1

Figure 2. Graph of logarithms of the relationship H/S of the sedimentary sequences of Lapao 5, Pastos Chicos and TC1 
ambientes más secos (Betancourt et al. 2000, Latorre et al. 2002). Las diferentes adaptaciones de estos dos tipos de plantas se reflejan en un gradiente vegetativo que cambia de una Puna dominada por césped en las zonas más húmedas, a un matorral xerófito con pocas gramíneas (Reese y Liu 2005). Por consiguiente, la relación H / A (todos expresados en logaritmos) sería 0 si los

Figura 3. Porcentaje (por locus y por materia prima) de: A. lascas de descortezamiento; $\mathrm{B}$. lascas de desbaste; $\mathrm{C}$. instrumentos

Figure 3. Percentage (by locus and raw material) of: A. preliminary flakes; $B$. knapping flakes; $C$. tools
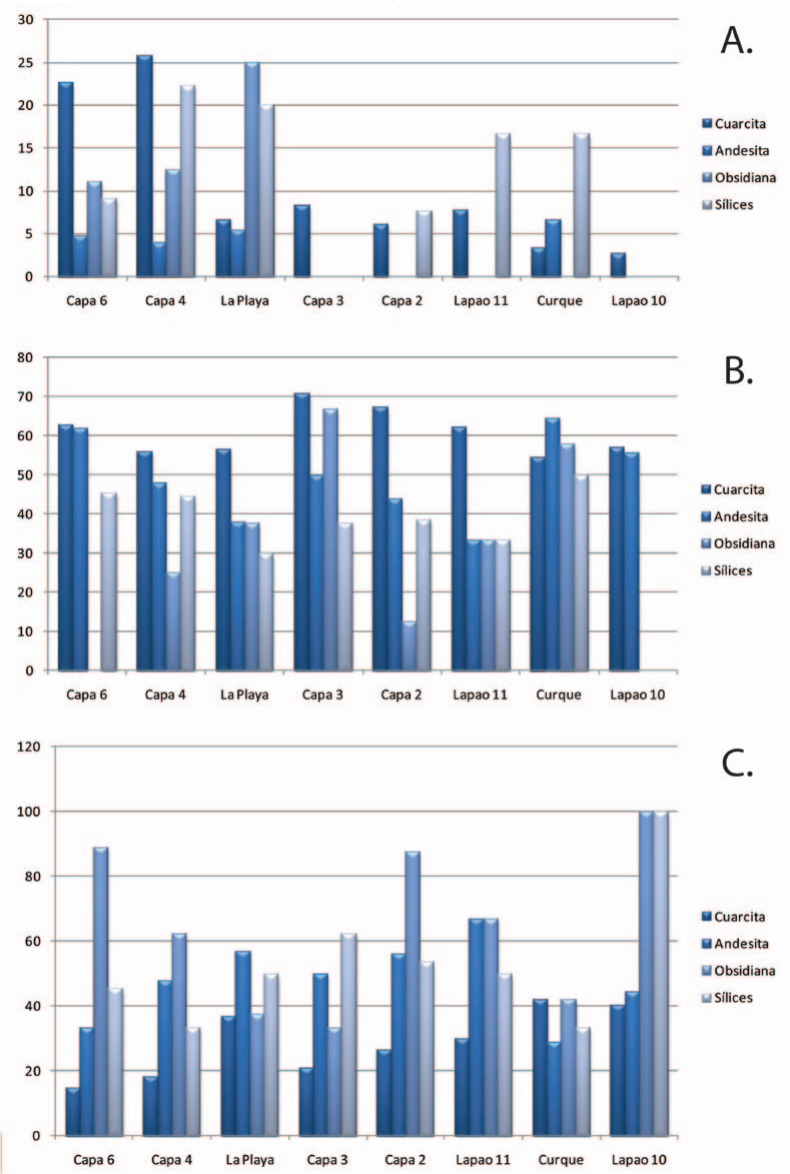

Tabla 5. Muestra total de los productos de talla relacionados a la formatización de los núcleos: lascas de descortezamiento, desbaste (formas base no retocadas y lascas de formatización de plataformas y superficies de talla), instrumentos (formas base retocadas o formatizadas)

Table 5. Total sample of knapping by-products linked to core reduction: preliminary flakes, knapping (non-retouched blanks and platforms and removal surfaces flaking), tools (retouched and shaped blanks)

\begin{tabular}{lccccccc}
\hline & Descortezamiento & $\%$ & Desbaste & $\%$ & Instrumentos & $\%$ & Total \\
\hline $\begin{array}{l}\text { Conjunto } \\
\text { capas 6 }\end{array}$ & 20 & 17,2 & 65 & 56,0 & 31 & 26,7 & 116 \\
Capa 4 & 21 & 19,4 & 55 & 50,9 & 32 & 29,6 & 108 \\
La Playa & 21 & 7,4 & 149 & 52,7 & 113 & 39,9 & 283 \\
Capa 3 & 2 & 4,1 & 30 & 61,2 & 17 & 34,7 & 49 \\
Capa 2 & 4 & 4,2 & 50 & 52,6 & 41 & 43,2 & 95 \\
Lapao 11 & 8 & 7,4 & 62 & 57,4 & 38 & 35,2 & 108 \\
Curque & 8 & 4,9 & 94 & 57,3 & 62 & 37,8 & 164 \\
Lapao 10 & 4 & 2,5 & 90 & 55,2 & 69 & 42,3 & 163 \\
\hline
\end{tabular}

porcentajes de polen de herbáceas/arbustivas son iguales. Los números positivos muestran la dominancia de pastos y por lo tanto condiciones más húmedas. Los valores negativos sugieren la dominancia de las Asteraceae sobre las gramíneas y por lo tanto condiciones más secas.

A partir del cálculo del índice de humedad (H/A) sobre las cuantificaciones polínicas de la secuencia Lapao 5 se distinguieron dos valores negativos que son interpretados como eventos de sequía. El primero de ellos hacia 9080 años AP y el otro hacia 8840 años AP (Figura 2). En los 2300 años que comprende la secuencia sedimentaria de Lapao 5 solo se detectan dos sequías, con una diferencia de 240 años entre una y otra. Asimismo, hay un valor cercano a cero $(0,04)$ hacia 8236 años $A P$, que también demuestra un importante descenso de los valores de humedad. Post 9080 años AP una recuperación rápida, mientras que post 8840 y 8236 años AP la recuperación es paulatina. La media calculada es de 0,52.

En el caso de Pastos Chicos el índice de humedad detectó solo una sequía hacia post 4200 años AP (Figura 2). El cálculo de la media demuestra que los Valores en general fueron altos en comparación con la secuencia de Lapao 5. Los picos más altos en 7583, 7252 y pre 4200 años AP. En promedio se detectó solo una sequía en ca. 5000 años AP.

A partir del índice de humedad se hallaron cuatro eventos de sequía: 11650 años AP, 5071 años AP, 4627 años AP y 4516 años $A P$, y dos valores cercanos a cero hacia 5738 años AP $(0,02)$ y 4891 años $\operatorname{AP}(0,06)$ años AP (Figura 2). La media calculada es baja en relación a los otros registros, se puede notar como esta baja post 5700 años AP. En términos generales se observan cuatro sequias en un tiempo de 8000 años AP. A simple vista se puede notar un aumento en la inestabilidad en los valores en relación a las otras secuencias analizadas, observándose en un aumento de media a 0,36.

\section{Aprovisionamiento de materias primas}

A partir del análisis de las actividades de desbaste (Tabla 5) se destaca una alta cantidad de lascas de descortezamiento en el conjunto de las capas 6 (ca. 9700 años AP) y 4 (ca. 8300 años AP) del alero Hornillos 2. Asimismo, los porcentajes de instrumentos son bajos para estas capas en relación a los otros locii. Sin embargo, se pudo detectar una marcada disminución de la representación de las lascas de descortezamiento en general, y particularmente para las cuarcitas durante la primera mitad del Holoceno medio (entre ca. 7800 y 6100 años AP) (Figura 3: A). 
Asimismo, se notó un incremento de las lascas de formatización de núcleos y formas base en obsidiana, llegando a su máximo valor en la capa 3 (Holoceno medio I, ca. 7300 años AP).

También en capa 2 se halló el porcentaje de lascas de desbaste más alto de toda la secuencia (Tabla 5), particularmente en cuarcita. Por otro lado, se observan altos porcentajes de lascas de desbaste en andesita durante el Holoceno temprano $(61,9 \%)$ y el Holoceno medio II, aunque debe señalarse la presencia de un núcleo de andesita en Lapao 11. Se destaca las bajas proporciones de lascas de desbaste en sílices parejas a lo largo de toda la secuencia, salvo en el caso de Curque (50\%) (Figura 3: B). En la capa 2 (ca. 6200 años AP) se encontró el único núcleo de obsidiana. También se hallaron tres núcleos de sílice en el alero Hornillos 2, las capas 6, 3 y 2.

El análisis de las proporciones de instrumentos en los
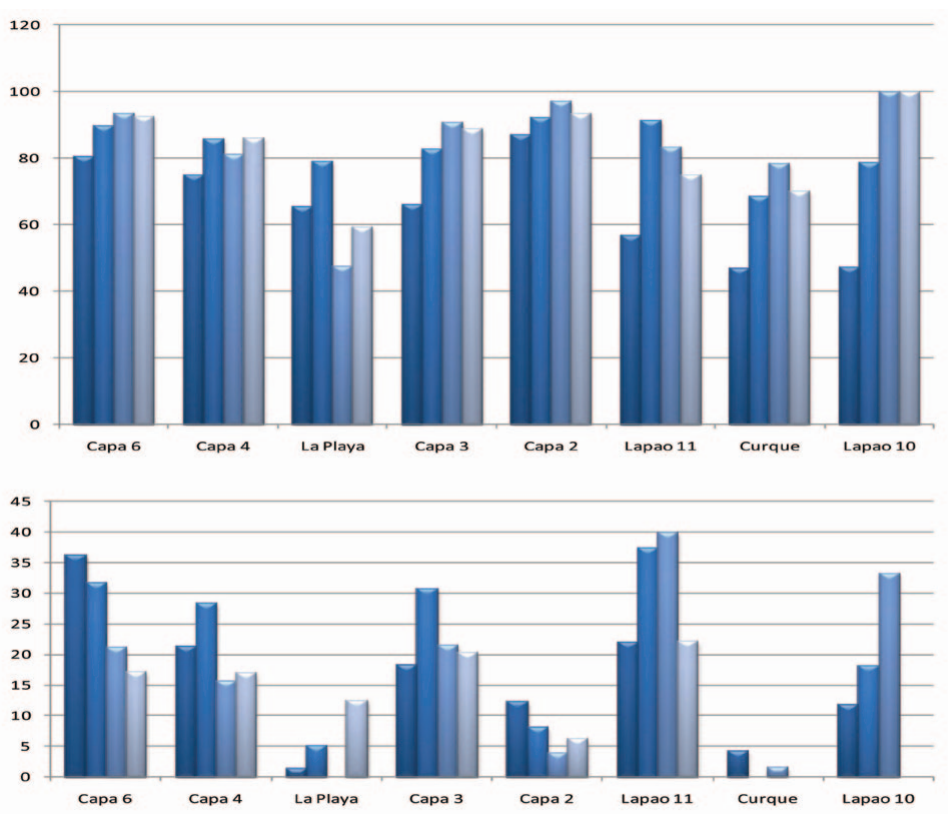

Figura 4. A. Lascas de formatización de instrumentos: B. Lascas de reactivación de instrumentos

Figure 4. Tool manufacture flakes; $B$. Tool resharpening flakes

\begin{tabular}{lccccr}
\hline & $\begin{array}{c}\text { Total } \\
\text { formatización de } \\
\text { instrumentos }\end{array}$ & $\%$ & $\begin{array}{c}\text { Total reactivación } \\
\text { de instrumentos }\end{array}$ & $\%$ & Total \\
\hline Conjunto capas 6 & 2316 & 60,9 & 671 & 17,6 & 3802 \\
Capa 4 & 1444 & 57,3 & 314 & 12,5 & 2520 \\
La Playa & 478 & 57,3 & 12 & 1,4 & 834 \\
Capa 3 & 468 & 47,4 & 103 & 10,4 & 987 \\
Capa 2 & 1849 & 79,7 & 146 & 6,3 & 2321 \\
Curque & 209 & 54,1 & 3 & 0,8 & 386 \\
Lapao 11 & 137 & 55,0 & 37 & 14,9 & 249 \\
Lapao 10 & 111 & 39,4 & 15 & 5,3 & 282 \\
\hline
\end{tabular}

Tabla 6. Lascas de formatización y de reactivación de instrumentos y total de la muestra (incluyendo todos los artefactos)

Table 6. Tool shaping and resharpening flakes and total sampling (including all artifacts)

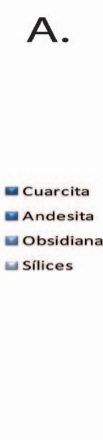

B.

conjuntos separados por rocas que mostró la dominancia de la obsidiana en la muestra durante el Holoceno prano y hacia la segunda mitad del Holoceno medio 3: C). Particularmente en el caso de las cuarcitas (Figura 3 : uso de instrumentos que se realizó en los sitios para la de núcleos y producción de formas base) en La Playa y en Hornillos 2 (capa 3). Al contrario, la proporción de instrumentos para cuarcita fue baja en general, pero tendió a crecer durante el Holoceno medio. Eso significaría las actividades de formatización de núcleo y de ucción de formas base en cuarcita fue importante en . presencia de núcleos de esta materia prima en todos los ocii corroboraría este hecho. Es posible que para el final del Holoceno medio (Curque, Lapao 10) la proporción relativamente alta de instrumentos para los conjuntos de cuarcita en relación a los otros locii, podría haber sido relacionada a una mayor integración de las formas base al equipamiento instrumental, tal como suele ocurrir con los desbastes de hojas presentes en estos sitios (Hoguin 2014). En efecto, la presencia de algunos núcleos ( $n=2)$, aunque baja, en Lapao 10 y relativamente alta en Curque $(n=8)$, demuestra que se realizaron actividades de desbaste en estos locii. Asimismo, a lo largo de la secuencia, la presencia de núcleos en cuarcita en todos los locii, y más particularmente en La Playa donde la frecuencia es relativamente alta también $(n=7)$, demuestra que se realizaron siempre estas actividades a lo largo del Holoceno, siendo la materia prima local. La proporción de instrumentos en los conjuntos de sílice tiende a crecer hacia ca. 7500 años AP (Hornillos 2, capa 3), y luego de bajar vuelve a subir hacia el final del Holoceno medio (Lapao 10).

En cuanto a las últimas secuencias que corresponden a la modificación de las formas base para confeccionar instrumentos, las lascas de formatización de instrumentos dominan el conjunto de desechos, aunque tienden a ser más bajas durante el Holoceno medio II (Tabla 6). Este hecho podría relacionarse con una menor formatización de instrumentos durante el Holoceno medio II, a diferencia de las actividades de desbaste que se encuentran más representadas durante este periodo (Tabla 5) y más 
particularmente en la cuarcita (Figura 4: A). Este fenómeno, en el que durante el Holoceno medio II las lascas de desbaste están mejor representadas que periodos anteriores, en contraposición a lo que ocurre con las lascas de formatización, que se encuentran menos representadas durante el Holoceno medio Il; ha sido observado en secuencias temporales relativamente largas donde los instrumentos y sus características funcionales están diseñadas en un primer tiempo con una mayor inversión técnica en la formatización de formas base, y luego una mayor inversión en la formatización de núcleos, cuya producción provee formas base que no requieren tanta modificación (Boëda 2013). Este hecho fue observado para esta secuencia (Hoguin 2013).

Es llamativa la baja proporción de lascas de reactivación en todas las materias primas para la capa 2 de Hornillos 2, La Playa, y Curque (Tabla 6, Figura 4: B); a diferencia de lo que se observa en las capas 6 de Hornillos 2 y en Lapao 11. Para la andesita, es notable la representación de estas lascas en el conjunto de capas 6, 4 y 3 de Hornillos 2 y en Lapao 11.

A modo de síntesis, es importante destacar ciertas tendencias. Para la cuarcita, a lo largo de la secuencia, se puede notar la predominancia de las actividades de desbaste en relación a las otras materias primas. Al contrario, observamos una predominancia de las actividades de confección de instrumentos para los conjuntos de sílices y las obsidianas. En los talleres, como La Playa, Curque, Lapao 10 (Holoceno medio II), predominan las actividades de desbaste en relación a los instrumentos descartados. De manera general, podemos observar cambios entre el Holoceno temprano (conjunto de capas 6 de Hornillos 2), el Holoceno medio I (capas 3 y 4 de Hornillos 2, La Playa) y el Holoceno medio II

\section{Regional (obsidianas)}

Holoceno temprano

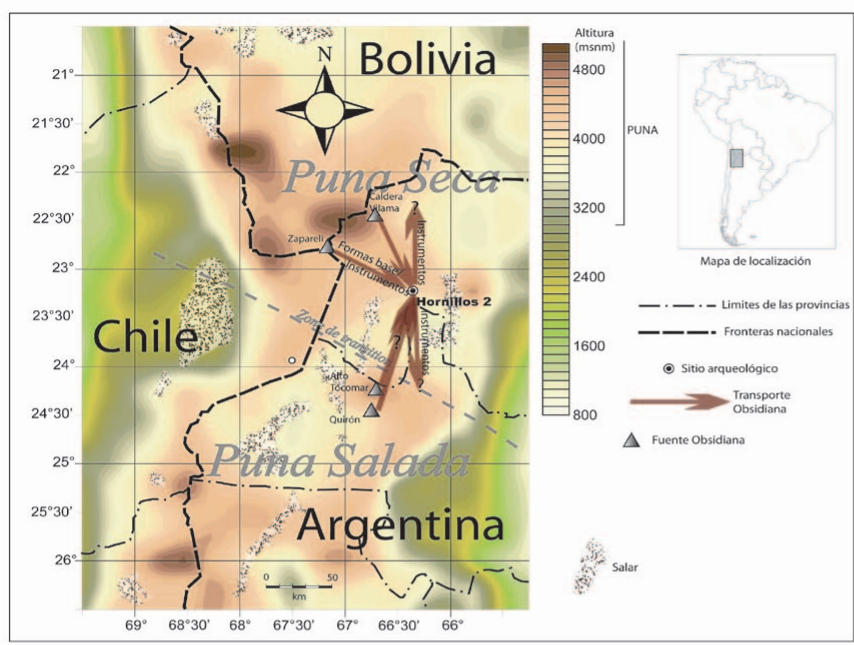

Holoceno medio

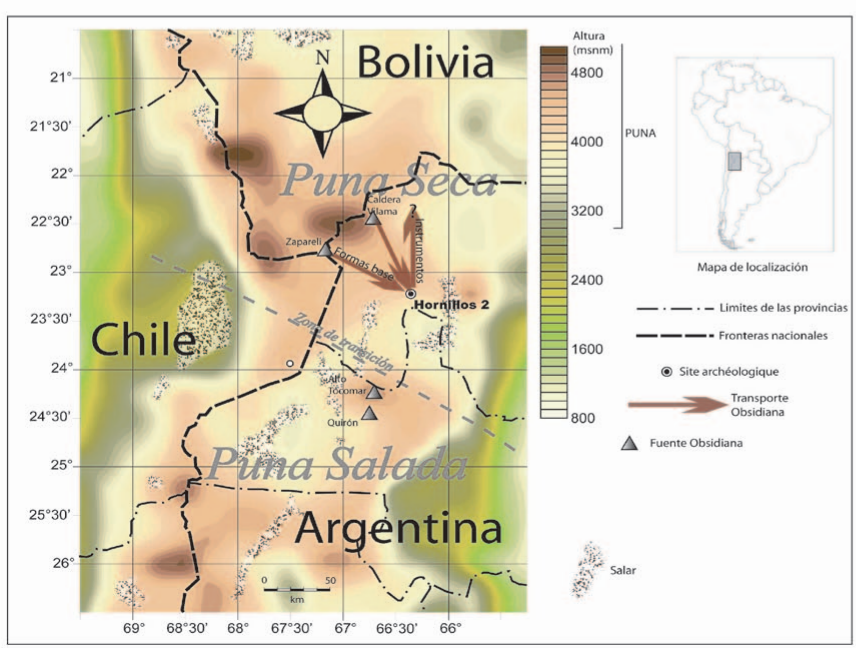

Local

Inicios

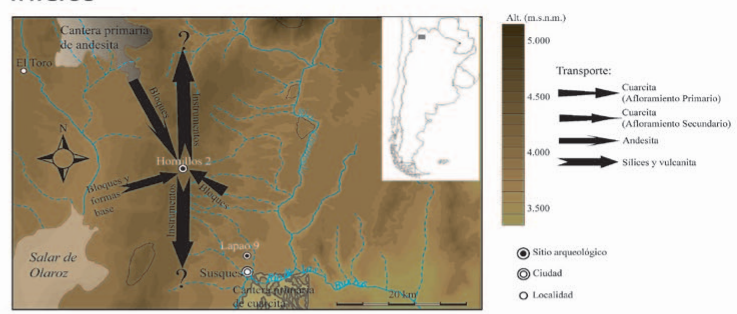

Transición Holoceno temprano-medio

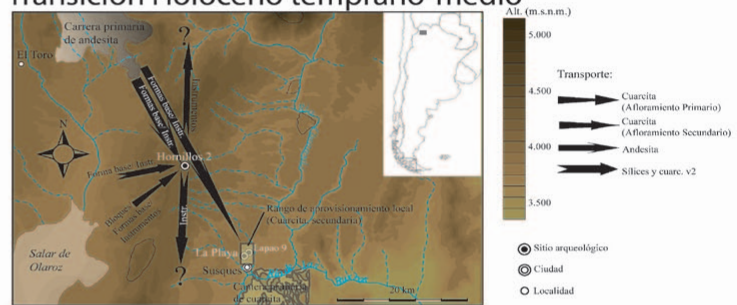

Holoceno medio I
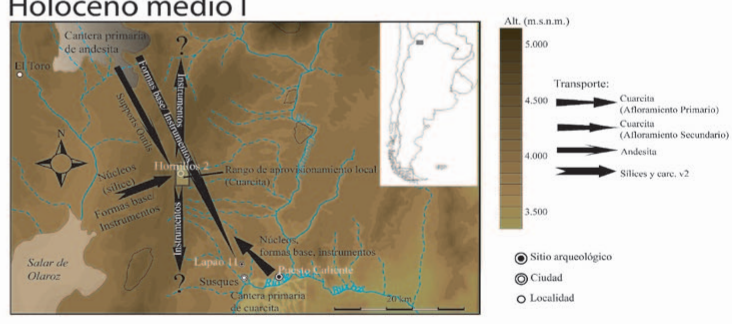

Holoceno medio II
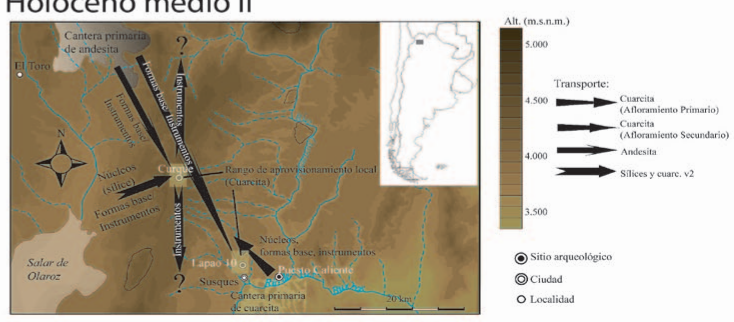

Figura 5. Mapas locales y regionales de los sitios y de los afloramientos de rocas aptas a la talla para el Holoceno temprano y medio

Figure 5. Local and regional maps of sites and sources of knappable rocks during Early and Mid Holocene 
(capa 2 de Hornillos 2, Lapao 10, Lapao 11 y Curque). Es importante destacar la importancia de las actividades de descortezamiento (particularmente para la cuarcita, pero también para la obsidiana y los sílices) para el Holoceno temprano y durante el inicio del Holoceno medio I.

\section{Discusión de los resultados}

\section{Pleistoceno final y Holoceno temprano}

Para la única muestra del Pleistoceno final obtenida de TC1 se ha podido observar el desarrollo a escala regional de una vegetación típica de ambientes más áridos que el actual, sin embargo a partir del análisis de la matriz sedimentaria se detecta que a nivel local se desarrolló un humedal, propio de un sistema estable de baja energía. Los antecedentes obtenidos en otras regiones como los análisis isotópicos y partículas de polvo en diferentes núcleos de hielo en los Andes (Thompson et al. 1995, Thompson et al. 1998, Ramírez et al. 2003), marcan un periodo más húmedo y frío. Por otra parte, los estudios realizados sobre columnas sedimentarias extraídas de diferentes cuencas lacustres señalan un primer momento más húmedo (13000 - 11500 años AP) seguido de otro más seco (Sylvestre et al. 1999, Baker et al. 2001, Bobst et al. 2001, Paduano et al. 2003).

Para el Holoceno temprano en Pastos Chicos no se registran sequias, sino más bien una vegetación estable típica de una estepa herbácea más húmeda que el ambiente actual. En tanto que en Lapao se han detectado dos eventos puntuales de sequía, con activación de la vega. En ambos casos se han observado condiciones generales de humedad durante el Holoceno temprano: entre 9300 - 8100 años AP en Lapao, y entre 9300 7000 años AP en Pastos Chicos. En líneas generales, se ha podido mostrar una mayor estabilidad de Pastos Chicos en relación al resto de las secuencias. Estas diferencias en la finalización del periodo de aumento de la humedad entre los registros de Lapao 5 y Pastos Chicos han sido explicadas como producto de las diferencias en el área de captación de la cuenca hídrica en la que se encuentra cada uno de ellos (Lapao $110 \mathrm{Km}^{2}$ y Pastos Chicos $1000 \mathrm{Km}^{2}$ ) (Tchilinguirian et al. 2014). En este marco ambiental más húmedo del Holoceno temprano, algunos indicadores, como la utilización de lajas de cuarcita local, nos mostró una intención de previsión en materias primas transportadas previamente. El aprovisionamiento de cuarcita parece haber consistido en excursiones en un radio de algunos kms alrededor del sitio Hornillos 2 (Figura 5). Asimismo, los bloques de cuarcita (disponibles localmente) estuvieron luego descortezados in situ en Hornillos 2 durante el Holoceno temprano. Si se consideran las lascas de descortezamiento como representativas del abastecimiento de bloques y de su traslado desde las fuentes hacia el sitio, se puede observar un cambio importante a partir del inicio del Holoceno medio (La Playa, capa 3 de Hornillos 2).
Hornillos 2 parece haber sido un campamento base durante el Holoceno temprano, donde se realizaron todas las secuencias de las cadenas operativas en la materia prima local (cuarcita) salvo en el caso de los sílices. El análisis de las materias primas no-locales (andesita y obsidiana) mostró principalmente actividades de formatización y de reactivación de instrumentos. Esto indicaría el traslado de instrumentos y/o formas base en el sitio para las materias primas no locales (Figura 5). Al contrario, para los sílices (locales pero de afloramientos escasos), varias formas base fueron seguramente producidas en el sitio y luego transportadas. Estos datos concuerdan con poblaciones de movilidad residencial alta y estrategias de tipo forager (sensu Binford 1980), realizando excursiones para distintas actividades alrededor de un sitio principal, transportando un equipamiento instrumental desde su precedente localidad, y un aprovisionamiento local con todas las actividades realizadas in situ. El hecho de llegar con sus implementos al sitio para realizar allí varias actividades fue también observado en la vertiente chilena (Herrera et al. 2015). Es también este tipo de estrategia y de movilidad la que pareció haber funcionado en la Puna Salada (Pintar y Rodríguez 2015), pero a diferencia de Antofagasta de la Sierra, los circuitos de movilidad de las poblaciones en la Puna Seca durante el Holoceno temprano habrían involucrado ocupaciones permanentes y exclusivamente puneñas, siguiendo un eje norte-sur (Yacobaccio et al. 2017). No se excluye tampoco un patrón de asentamiento con movilidad logística, tal como fue propuesto para las tierras altas en el norte de Chile (Osorio et al. 2017). Sin embargo, los cambios observados para el Holoceno medio, mostrarían una disminución de la movilidad en general.

\section{Holoceno medio I y II}

Para el Holoceno medio el índice de humedad basado en los cambios composicionales de la vegetación marcan una sola sequía en el registro de Lapao 5 y tres en el Testigo Cruces 1 de Barrancas. Sin embargo, también se debe mencionar que al estudiar el espectro polínico completo, se ha observado que en ambas secuencias bajo condiciones de aridez regional se desarrollan vegas. Es interesante señalar que los antecedentes coinciden en marcar que cuando las condiciones de aridez regional aumentan, las vegas por debajo de la cota de $4100 \mathrm{~m}$ snm se activan y se expanden. Esto se debe a la baja energía del aporte de agua de la cuenca que hace que el agua se encharque y se mantenga estable permitiendo el desarrollo de la vegetación típica de vega (como Myriophyllum, Pteridophyta y Cyperaceae). De todas formas, dentro del bloque temporal del Holoceno medio se puede hacer una diferenciación. Según lo observado en la secuencia de TC1 en Barrancas post 5800 años AP baja considerablemente la media del índice de humedad, por lo que se pueden advertir dos momentos: uno más húmedo entre 6400-5860 años AP y otro más seco entre 5860-4071 años AP. Incluso, dentro del periodo más seco, se puede notar un momento aún más marcado de 
Figura 6. Síntesis de los índices de humedad aplicados a los tres registros de polen bajo estudio (TC1, L5 y PCH), cuya cronología abarca el periodo 11671-4071 años AP

Figure 6. Synthesis of humidity indices applied to the three pollen records under study (TC1, L5 and PCH), whose chronology covers the period 11671-4071 years BP

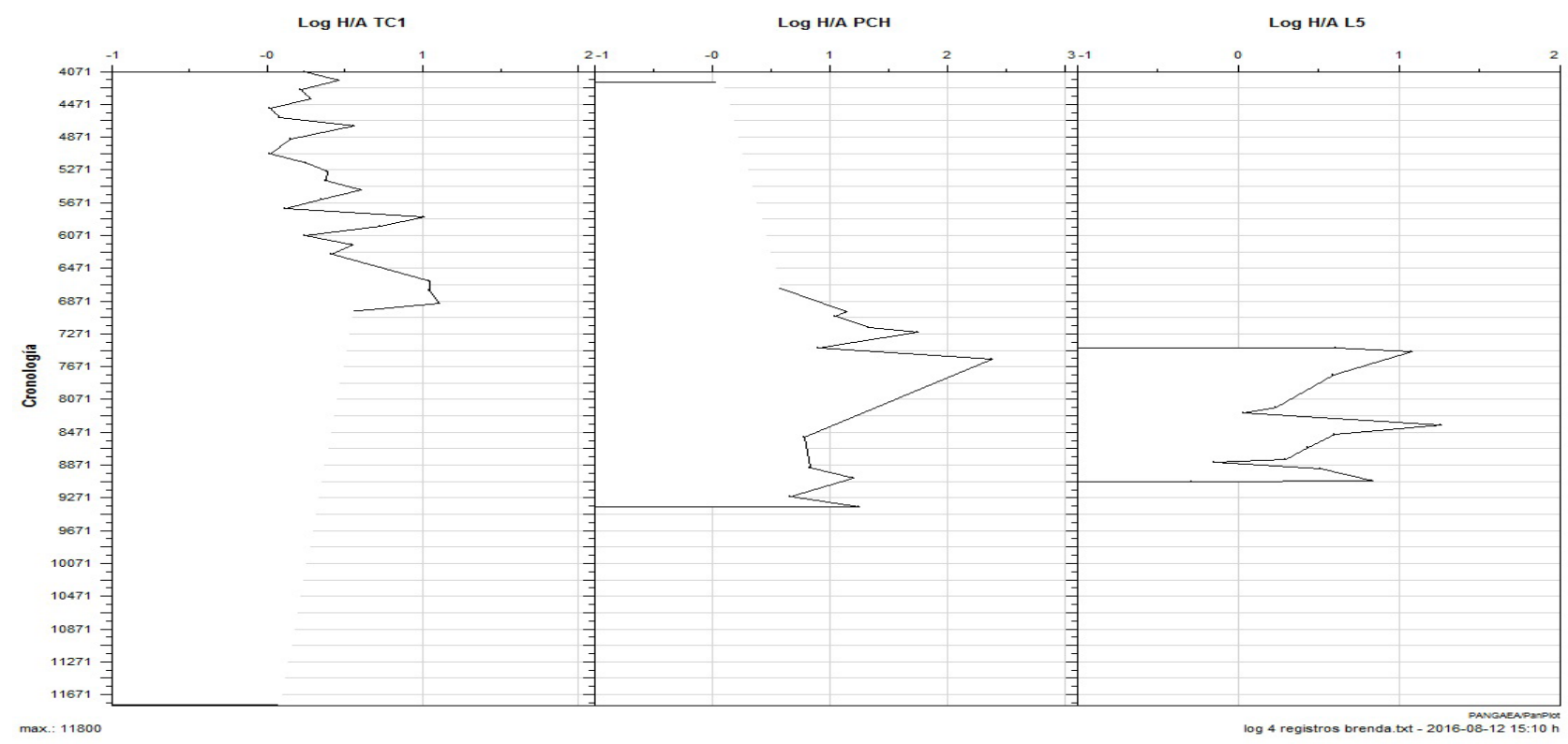

aridez post 5000 años AP. Estos resultados coinciden con las tendencias generales que muestran un proceso de aridez durante el Holoceno medio, acentuándose hacia la segunda mitad de este periodo, en los Andes Tropicales y en la Puna Argentina. Aunque algunas localidades parecen haber retenido condiciones de humedad local (Tchilinguirian y Morales 2013). Las diferentes respuestas de estos ambientes locales a los cambios ambientales de mega escala $\left(5.1 \times 10^{8} \mathrm{Km}^{2}\right.$ (según Dincause 2000$)$ pueden deberse, al igual que en el caso del Holoceno temprano, a las características de la cuenca. Sobre las posibles causas de los cambios ambientales detectados durante el Holoceno medio, se sostiene que un aumento de las temperaturas podría haber producido el aporte de agua de deshielo en cotas más bajas, dando como resultado un aumento del nivel de lagos en algunas localidades de la Puna (Rech et al. 2002; 2003, Grosjean et al. 2001). Coincidentemente con la diferenciación observada dentro del periodo del Holoceno medio, Morales (2011) a partir del relevamiento de los antecedentes regionales de depósitos de turbas hace una diferenciación entre lo que él Ilama el Holoceno medio 1 (8000-6000 años AP) y 2 (6000-4500 años AP), siendo este último aún más árido. Estas observaciones cobran fundamental relevancia para discutir la variabilidad del registro arqueológico dentro de este periodo (Figura 6).

Salvo a principios del Holoceno medio (correspondiente a la capa 4 del alero Hornillos 2), en general se observó que no todas las cadenas operativas se desarrollaron en los sitios (salvo también en La Playa donde abundan los productos correspondiendo a distintas etapas de las cadenas operativas). En términos generales, los núcleos llegaron descortezados en los sitios, salvo en la capa
4 de Hornillos 2, que parece reflejar una continuidad con el Holoceno temprano. Esta tendencia de que los núcleos lleguen a los sitios descortezados, o sea que las actividades de descortezamiento se realizan fuera de los sitios estudiados (salvo para el Holoceno temprano y para la capa 4 de Hornillos 2 (que acá lo mencioné como siendo del inicio del Holoceno medio), se ve acentuada durante la segunda mitad del Holoceno medio. Más particularmente, en la capa 3, la baja cantidad de materiales, la alta proporción de puntas de proyectil y el estado agotado de los núcleos nos lleva a pensar que el sitio Hornillos 2 fue especializado en ciertos tipos de actividades para esta ocupación, y que solamente las últimas etapas de las cadenas operativas se desarrollaron allí (Hoguin y Yacobaccio 2012). Esto coincidiría con una ocupación de tipo logístico para el alero en este período. Por otro lado, se observó también un marcado cambio a partir de la capa 4, registrando mayor proporción de lascas de descortezamiento para los sílices (Figura 3: A). Dadas las características de los afloramientos de estas rocas (filones muy acotados y poco accesibles en el paisaje), es probable que su explotación requiera un buen conocimiento del ambiente. Las tendencias así observadas podrían estar relacionadas con un mayor conocimiento del ambiente geológico de parte de las poblaciones a partir del Holoceno medio, hecho que fue observado en Antofagasta de la Sierra a partir de estudios de procedencia de las obsidianas (Pintar et al. 2016). Sin embargo, se debe aclarar que, al contrario de los niveles más antiguos, los análisis geoquímicos de las obsidianas de esta capa, y de la siguiente (capa 2) mostraron una procedencia exclusiva de los afloramientos del norte de la región (Figura 5), posiblemente reflejando un cambio importante en los circuitos de movilidad (Yacobaccio et 
al. 2017). Este cambio se vio acompañado de una mayor cantidad de artefactos en obsidiana en la capa 3 del alero Hornillos 2, siendo la materia prima la más representada de toda la secuencia.

Para la andesita, se destacó la importancia de los instrumentos para el Holoceno medio I (8500-6200 años AP) y de las lascas de desbaste para el Holoceno medio II (6200-3500 años AP). Esto mostraría un aprovisionamiento planificado en formas base e instrumentos obtenidos en otra localidad o producidos por núcleos circulando a través varios sitios (Figura 5).

Para el Holoceno medio II, durante el cual se acentuó el proceso de aridización, se observó cierta especialización de los sitios en función de las actividades de talla y del abandono de los instrumentos (Figura 5). En efecto, en Lapao 11, se pudo observar que los pocos núcleos de cuarcita no procedían de los alrededores inmediatos del sitio, o incluso de la quebrada. Al contrario, en el sitio Puesto Caliente, solamente se constató la presencia de núcleos (Hoguin 2013). El único sitio donde una proporción relativamente significativa de puntas de proyectil fue hallada fue en Hornillos 2 (capa 2). Los instrumentos, estuvieron presentes tanto en Hornillos 2 como en Lapao 11. Se pudo observar una gestión particular de la materia prima, porque los núcleos parecieron estar transportados para que algunas operaciones de desbaste se realicen en los campamentos. Aunque en todos los casos, ciertas actividades de desbaste debieron realizarse localmente, considerando que la cuarcita está presente en todo el área de estudio, en ambos sitios un aporte bajo de formas base, de preformas (particularmente en el caso de Lapao 11) y de núcleos previamente descortezados circularon permitiendo aprovisionar los sitios. Esto pareció ser el caso de la andesita, mostrando la misma importancia en relación al periodo anterior. Hornillos 2 parece ser un campamento donde se desarrollaron varias actividades, al contrario de Lapao 11.

Es relevante observar como el porcentaje de lascas de desbaste en todas las materias primas, pero particularmente para la andesita, tendió a crecer durante la segunda mitad del Holoceno medio. Es notable el alto porcentaje de lascas de desbaste así como de las de formatización de instrumentos. De esta manera, parece probable que los núcleos hayan circulado ya descortezados y preparados para la extracción de formas base en los sitios analizados. Confirmaría la propuesta que a partir del Holoceno medio, hubo una especialización tecnológica de los sitios, algunas actividades como el descortezamiento, la extracción de formas base desarrollándose prioritariamente en ciertos sitios. Estas estrategias se pudieron dar posiblemente en el marco de una movilidad más baja (en relación al Holoceno temprano) y más de tipo logística (es decir con sitios especializados).
Para finales del Holoceno medio, los núcleos llegaron también descortezados en los sitios analizados, salvo quizás en el caso de las rocas silíceas. Estas etapas de descortezamiento probablemente se desarrollaron en los afloramientos primarios, en sitios como Puesto Caliente (Figura 5) donde se observó también la presencia importante de núcleos de distintos tamaños (Hoguin 2013). Se observa un equilibrio entre las proporciones de instrumentos en relación a los otros productos del desbaste, particularmente en el caso de la cuarcita y de la andesita. Esto pareciera resultar por la economía del desbaste, laminar en este caso (Hoguin 2014). Para estas materias primas, las actividades de desbaste fueron relativamente importantes en los sitios de la Quebrada de Lapao, con una integración en instrumentos de los productos obtenidos. Esto podría estar relacionado con el fenómeno de que en secuencias de ocupación largas, en un primer momento los instrumentos tienen mayor inversión en las secuencias de formatización (retoque, adelgazamiento, retalla, etc.) de las formas base obtenidas por los métodos más simples (núcleos con un par de lascados); Luego ocurre que estos mismos instrumentos (pueden ser puntas, cuchillos, lo que sea) muestran la mayor inversión de trabajo en la obtención de la forma base y que posteriormente no requiere tanta formatización (Boëda 2013). A pesar de esto, estos datos estarían en relación con un aprovisionamiento de las materias primas que involucró una selección de bloques específicos, que podrían ser preparados previamente y llegar a los sitios para proveer las formas base (Figura 5). Para finalizar, es importante indagar sobre el hecho que los requerimientos tecnológicos son un aspecto importante de las estrategias de aprovisionamiento de materias primas, no siempre relacionadas con la movilidad (Restifo 2017).

\section{Conclusiones}

Según la cronología, las distintas etapas de confección, uso y descarte de instrumentos líticos no se desarrollaron en las mismas localidades. Sin embargo, es necesario tomar recaudos en relación a la interpretación de los presentes análisis, ya que las localizaciones investigadas no representan la totalidad de sitios utilizados por grupos cazadores-recolectores para sus estrategias de aprovisionamiento y sus actividades de talla.

A partir de los resultados expuestos se pueden delinear las siguientes conclusiones:

- Durante el Holoceno temprano las condiciones ambientales fueron más húmedas y estables que en la actualidad. Las características de la vegetación se asemejan a las de un pastizal de altura como los que hoy se encuentran en cotas más altas. La extensión del pastizal de altura a cotas más bajas habría producido una disminución la distancia entre los parches productivos de vegetación. El registro arqueológico analizado, junto a los antecedentes conocidos para el área, permiten 
sostener que bajo esta estructura más productiva (productividad primaria y secundaria) de recursos más abundantes y homogéneamente dispersos en el paisaje, los grupos humanos de cazadores-recolectores habrían desarrollado una estrategia de alta movilidad, con un aprovisionamiento directo de ciertas materias primas, con bases residenciales donde se realizaron la totalidad de las secuencias de talla para las materias primas locales, y donde se trasladaron instrumentos reactivados o reciclados en materias primas distantes, tal como ha sido mencionado en otros sitios (Herrera et al. 2015)

- Durante el Holoceno medio I los análisis polínicos, en concordancia con los antecedentes paleomabientales, señalan una baja paulatina en la media de humedad, un aumento en la distancia entre los parches de vegetación, pero con el desarrollo de vegas en espacios más acotados del espacio y con una alta productividad primaria. Estas características ambientales habrían propiciado un cambio hacia una estrategia de movilidad de tipo logística y el uso de sitios más específicos (tanto desde el punto de vista de la tecnología lítica como de otras actividades), una planificación de las actividades de talla con circulación de núcleos descortezados y preparados en las canterastalleres y luego su traslado y circulación en varios sitios para aprovisionarlos en formas base e instrumentos.

- Durante el Holoceno medio II, y según el índice de humedad aplicado a la vegetación, el marco ambiental se torna aún más árido. Esto produce una mayor fragmentación del ambiente. Asimismo, se detecta un importante aumento en la frecuencia de las variaciones ambientales. Estas variaciones podrían haber fomentado también un cambio en las estrategias adaptativas de los grupos humanos hacia una especialización más marcada de los sitios. Asimismo, los cambios observados durante el Holoceno medio (I y II) podrían estar relacionados a una movilidad menor, y un crecimiento del tamaño de los grupos, sustentado por la domesticación de camélidos, usando pasturas aptas a sustentar rebaños para esta estrategia.

\section{Agradecimientos}

A Hugo Yacobaccio, Liliana Lupo y Eric Boëda por la dirección de los proyectos de investigación. A la financiación del proyecto UBACYT 230BA y CONICET PIP 0569. A los dos evaluadores de este trabajo por sus comentarios que permitieron mejorar el manuscrito original.

\section{Bibliografía}

Aguerre, A., Fernández Distel, A., Aschero, C. A. 1975. Comentarios sobre nuevas fechas en la arqueología precerámica de la Provincia de Jujuy. Relaciones de la Sociedad Argentina de Antropología, Tomo IX: 211-214.

Aldenderfer, M. 1998. Montane Foragers. Asana and the South-Central Andean Archaic. University of Iowa Press, 327p.

Aschero, C. 1994. Reflexiones desde el Arcaico Tardío (6000-3000 A.P). Rumitana 1: 13-17.

Aschero, C., J. Martínez. 2001. Técnicas de caza en Antofogasta de la Sierra. Relaciones de la Sociedad Argentina de Antropología Tomo XXVI: 215-241.

Baker, P., G. Seltzer, S. Fritz, R. Dunbar, M. Grove, P. Tapia, S. Cross, H. Rowe, J.Bobst, A., T. K. Lowenstein, T. E. Jordan, L. V. Godfrey, M. C. Hein, T. L. Ku y S. Betancourt, J. L., C. L. Quade y K. A. Rylander 2000. 22000 BP years record of monsoonal precipitation from nortern Chile's Atacama desert. Science 289: 1542-1546.

Boëda, E. 2013. Techno-logique et technologie. Une paléo-histoire des objets lithiques tranchants. @rchéoéditions.com.

Bousman, B. 1993. Hunter-Gatherer adaptation, Economic risk and tool Design. Lithic Technology 18: 59-86.

Bustillo, M. A., Alonso, R. 1989. Nódulos opalinos en facies marginales del Salar de Olaroz (Puna argentina). Estudios Geológicos 45: 55-59.

Cabrera, A. 1976. Regiones fitogeográficas Argentinas. En Enciclopedia Argentina de Agricultura y Jardinería: 1-85. ACME, Buenos Aires.

Dincauze, D. 2000. Environmental Archaelogy: Principles and Practice. Cambridge University Press, London.

Faegri, K., J. Iversen 1989. Textbook of pollen analysis. John Wiley \& Sons. Chichester, [4ta. Edición a cargo de K. Fægri, P. E. Kaland y K. Krzywinski]

Fernández, J., V. Markgraf, H. Panarello, M. Albero, M. Angiolini, S. Valencio y M. Arriaga.1991. Late Pleistocene/ Early Holocene Environments and climates, Fauna, and Human occupation in the Argentine Altiplano. Geoarchaeology: An International Journal 6 (3): 251-272.

Fernández Distel, A. 1974. Excavaciones Arqueológicas en la Cueva de Huachichocana, Dep. de Tumbaya, Prov. de Jujuy, Argentina. Relaciones de la Sociedad Argentina de Antropología Tomo VIII: 101-127.

Grosjean, M., Van Leeuwen, J.N., Ammann, B., Geyh, M.A., Van Der Knaap, W.O., Tanner, W. 2001. A 22,000 year sediment and pollen record from Laguna Miscanti, northernChile, CentralAndes 24 S.Global and Planetary Change 28:35-51

Halstead, P. y J. O'Shea. 1989. Introduction: cultural 
responses to risk and uncertainty. En P. Halstead y J. O'Shea (eds.), Bad Year Economics: 1-7. Cambridge, Cambridge University Press.

Hernández Llosas, I. 2000. Quebradas altas de Humahuaca a través del tiempo: el caso de Pintoscayoc. Estudios Sociales del N.O.A (4) 2: 167- 224.

2005. Pintoscayoc and the archaeology of the arid puna and rift valley, northern Argentina. En Smith, S., \& Hesse, P. (Eds), 23 South, Archaeology and Environmental History of the Southern Deserts (págs. 186-197). Canberra: National Museum of Australia Press.

Herrera, K. A., Ugalde, P. C., Osorio, D., Capriles, J. M., Hocsman, S., Santoro, C. M. 2015. Análisis tecnotipológico de instrumentos líticos del sitio arcaico temprano Ipilla 2 en los Andes de Arica, Chile. Chungará, Volumen 47, N¹: 41-52.

Herrera, K. A., Sepúlveda, M., Gayo, E. M., Latorre, C., Jackson, D., De Pol-Holtz, R., Santoro, C. M. 2017. Hunter-Gatherer Mobility Strategies in the High Andes of Northern Chile during the Late-Pleistocene-Early Holocene Transition. Journal of Field Archaeology, Vol. 42, No. 3: 228-240.

Heusser, C. J. 1971. Pollen and Spores of Chile. Modern types of the Pteridophyta, Gymnospermae and Angiospermae. Tucson, the University of Arizona Press.

Hogg, A. G., Q. Hua, P. G. Blackwell, M. Niu, C. E. Buck, T. P. Guilderson, T. J. Heaton, J. G. Palmer, P. J. Reimer, R. W. Reimer, C. S. M. Turney y S. R. H. Zimmerman. 2013. SHCal13 Southern Hemisphere Calibration, 0-50,000 Years cal BP. Radiocarbon, 55(4): 1889-1903.

Hoguin, R. 2013. "Evolution et changements techniques dans les sociétés de chasseurs-cueilleurs de la Puna Sèche des Andes Centrales-Sud: Technologie lithique dans la localité de Susques durant I'Holocène ancien et moyen". Thèse de doctorat en co-tutelle Universidad de Buenos Aires, Université de Paris X. Unversidad de Buenos Aires, Facultad de Filosofía y Letras.

2014. La tecnología de hojas durante el Holoceno medio en la Puna oriental de Atacama: El caso de Susques (Provincia de Jujuy, Argentina). En M. Farias, $\&$ A. Lourdeau (Dir.), Poblamiento de América del sur: la contribución de la tecnología lítica (págs. 221-253). @ archéo éditions.

2015. Secuencia cronológica y Tecnología lítica en la Puna Seca de los Andes Centro-Sur para el Holoceno temprano y medio a través el ejemplo de Susques. Relaciones de la Sociedad de Antropología Argentina Tomo XXXIX: 333-364.
Hoguin, R., Yacobaccio, H. 2012. Análisis lítico de ocupaciones del Holoceno medio de Hornillos 2 (Jujuy, Argentina): Discutiendo la tecnología y distribución de las puntas de proyectil "San Martín". Chungará Volumen 44, No 1: 81-95.

Inizan, M.L., Reduron, M., Roche, H., \& Tixier, J. 1995. Technologie de la pierre taillée. Tome 4. Meudon: CREP.

Jackson, D., Méndez, C., De Souza, P. 2004. Poblamiento Paleoindio en el norte-centro de Chile: Evidencias, problemas y perspectivas de estudio. Complutum Vol. 15: $165-176$.

Joly, D., Santoro, C. M., Gayo, Ugalde, P. C., March, R. J., Carmosa, R., Marguerie, D., Latorre, C. 2017. Late Pleistocen fuel management and human colonization of the Atacama Desert, northern Chile. Latin American Antiquity 28 (1): 144-160.

Kent, S. 1992. Studying variability in the archaeological record: An ethnoarchaeological model for distinguishing mobility patterns. American Antiquity 57: 635-660.

Kulemeyer, J., L. Lupo. 1994. Evolución de paisaje bajo influencia antrópica durante el Holoceno Superior en la cuenca del río Yaví. Borde Oriental de la Puna. Jujuy, Argentina. Bamberg SüdamerikaSymposium. Landschaftsentwicklung, Paläoökologie und Klimageschichte der Ariden Diagonale Südamerikas im Jungquartär.

Latorre, C., J. L. Betancourt, K. A. Rylander y J. Quade. 2002. Vegetation invasions into absolute desert: a 45 $000 \mathrm{yr}$ rodent midden record from the Calama-Salar de Atacama basins, northern Chile (lat 22-24S). Geological Society of America Bulletin 114: 349-366.

Liu, K. B., C. A. Reese, L. G. Thompson. 2005. Ice-core pollen record of climatic changes in the central Andes during the last 400 yr. Quaternary Research 64(2): 272278.

López, G. E. J. 2008. Arqueología de Cazadores y Pastores en Tierras Altas: Ocupaciones humanas a lo largo del Holoceno en Pastos Grandes, Puna de Salta, Argentina. Oxford: BAR International Series.

López, G. J. E., Orsi, J. P. 2017. El sitio Abrigo Pozo Cavado, Puna de Salta. Nuevo aporte arqueológico y cronológico para el estudio de procesos de cambio hacia finales del Holoceno medio (ca. 5600-4600 años AP). Revista del Museo de Antropología 10 (1): 43-48.

Loyola, R., Cartagena, I., Núñez, L., López, M. P. 2017. Moving into an arid landscape: Lithic technologies of the Pleistocene-Holocene transition in the high-altitude bassins of Imilac and Punta Negra, Atacama Desert. 


\section{Quaternary International: En prensa.}

Lupo, L. 1998. "Estudio sobre la lluvia polínica actual y la evolución del paisaje a través de la vegetación durante el Holoceno en la cuenca del río Yavi. Borde Oriental de la Puna, Noroeste Argentino". Tesis Doctoral inédita, Fakultat fur Geschichts-und Geowissenschaften, Universitat Bamberg.

Markgraf, V. 1985. Paleoenvironmental History of the last 10000 years in Northwestern Argentina. Zentralblatt Geologie und Palantologie. Teil I: 1739-1749.

Markgraf, V., H. D' Antoni. 1978. Pollen Flora of Argentina. Tucson, University of Arizona Press.

Maro, G., Caffe, P. J. 2012. Volcanismo terciario de la Puna jujeña, los Cerros Negros de Jama. Aportes al Magmatismo y Metalogénesis Asociada I. Serie Correlación Geológica 28(1): 51-72.

Martínez, J. 2003. Ocupaciones humanas tempranas y tecnología de caza en la microregion de Antofagasta de la Sierra (10 000-7000 AP). Tesis doctoral. Facultad de Ciencias Naturales, Instituto Miguel Lillo. Universidad Nacional de Tucuman, Argentina.

Martínez, J. 2012. Evidence of Early Human Burials in the Southern Argentinian Puna. Current Research of the Pleistocene. Southbound, Late Pleistocene Peopling of Latin America. Special Edition: 75-78.

Martínez, J. M., Mauri, E. P., Mercuri, C., Caria, M. A., Oliszewski, N. 2013. Mid-Holocene occupations in Tucumán (northwest Argentina). Quaternary International 307: 86-95.

Mercuri, C., Restifo, F. 2014. Application of PhysicoChemical and Macroscopic Methods to Lithic Artifacts Studies from Alero Cuevas site (Salta, República Argentina): A Complementary Approach. En D. M. Kligmann, M. R. Morales editores, Physical, Chemical and Biological Markers in Argentine Archaeology: Theory, Methods and Applications (pág. 27-39). London: BAR Archaeological Series.

Minc, L.D., Smith, K.P. 1989. The spirit of survival: cultural responses to resource variability in north Alaska. In: Halstead, P., O'Shea, J. (Eds.), Bad Year Economics: Cultural Responses to Risk and Uncertainty. Cambridge University Press, Cambridge, pp. 8-39.

Mondini, M., Martínez, J. G., Pintar, E., Reigadas, M. C. 2013. Middle Holocene foraging, mobility and landscape use in the southern Argentinean Puna: Hunter-gatherers from Antofagasta de la Sierra, Catamarca, Argentina. Quaternary International 307: 66-73.
Morales, M. 2011. Arqueología ambiental del Holoceno Temprano y Medio en la Puna Seca argentina Modelos paleoambientales multi-escalas y sus implicancias para la arqueología de cazadores-recolectores. Oxford, BAR S2295, South American Archaeology Series 15, Archaeopress. 241 pp.

Morales, M., Bustos, S.; Oxman, B., Pirola, M., Tchilinguirian, P., Orgeira, MA. J., Yacobaccio, H. 2016. Exploring habitat diversity of Mid-holocene huntergatherers in the tropical Andes: multiproxy analysis of cruces core 1 (TC1) Dry Puna of Jujuy, Argentina. Journal of Archaeologial Science: Reports. En prensa.

Muscio, H. 1998-1999. Tendencias en la variabilidad ambiental de la Puna Argentina: Implicancias para la ecología humana prehistórica y para los paisajes arqueológicos. Cuadernos del Instituto Nacional de Antropología y Pensamiento Latinoamericano 18: 271296.

Muscio, H., López, G. 2017. Radiocarbon dates and anthropogenic signal in the South-Central Andes (12,500600 cal. years BP). Journal of Archaeological Science 65: 93-102.

Osorio, D., Steele, J., Sepúlveda, M., Gayo, M. E., Capriles, J. M., Herrera, K., Ugalde, P., De Pol-Holz, P., Latorre, C., Santoro, C. M. 2017. The Dry Puna as an ecological megapatch and the peopling of South America: Technology, mobility, and the development of a late Pleistocene/early Holocene Andean hunter-gatherer tradition in northern Chile. Quaternary International 461: 41-63.

Oxman, B. 2015. "Paleoambiente y sociedad durante le Holoceno en la Puna de Jujuy: un abordaje arqueopalinológico". Tesis doctoral, Facultad de Filosofía y Letras, UBA.

Oxman B., H. Yacobaccio. 2014. Paleoenvironmental and archeological implications during the Holocene in the Dry Puna of Argentina. En D. M. Kligmann y M. R. Morales (eds.), Physical, Chemical and Biological Markers in Argentine Archaeology: Theory, Methods and Applications: 105-116. Oxford, BAR International Series 2678.

Paduano, G. M., M. B. Bush, P. A. Baker, S. C. Fritz, y G. O. Seltzer. 2003. A Vegetation and Fire History of Lake Titicaca since the Last Glacial Maximum. Papers in the Earth and Atmospheric Sciences. Paper 36.

Pintar, E. 1995. Los conjuntos líticos de los cazadores Holocénicos en la Puna Salada. Arqueología 5: 9-24.

Pintar, E. 2014. Continuidades e hiatos ocupacionales durante el Holoceno medio en el borde oriental de la Puna Salada, Antofagasta de la Sierra, Argentina. Chungara, 
Volumen 46, №1: 51-71.

Pintar, E., Rodríguez, M. F. 2015. Undersanting foraging radius and mobility in a high desert. Journal of Archaeological Science Vol. 59: 142-158.

Pintar, E., Martínez, J. G., Aschero, C. A., Glascock, M. D. 2016. Obsidian use and mobility during the Early and Middle Holocene in the Salt Puna, NW Argentina. Quaternary International 422: 93-108.

Pirola, M. 2014. “¿Dónde va la gente cuando no llueve? Heterogeneidad ambiental multi-escala en la Puna de Jujuy durante el Holoceno Medio: contenido de materia orgánica y carbonatos en sedimentos de paleohumedales". Tesis de Licenciatura. Facultad de Filosofía y Letras. Universidad de Buenos Aires.

Ramirez, E., G. Hoffman, J. D. Taupin, B. Francou, P. Ribstein, N. Caillon, F. A. Ferron, A. Landais, J. R. Petit, B. Pouyaud, U. Schotterer, J. Simoes y M. Stievenard. 2003. A new Andean deep ice core from Nevado Illimani (6350 m), Bolivia. Earth and Planetary Science Letters 212: 337-350.

Ratto, N. 2003. “Estrategias de Caza y Propiedades del Registro Arqueológico en la Puna de Chaschuil (Dpto. Tinogasta, Catamarca, Argentina). Buenos Aires". Tesis Doctoral, Universidad de Buenos Aires, Facultad de Filosofía y Letras.

Rech, J., E. Quade, J. Betancourt. 2002. Late quaternary paleohydrology of the Central Andes (22- 24\% $\mathrm{S}$ ), Chile. Geological Society of America Bulletin 114: 334-348.

Rech, J., S. Pigati, J. Quade y J. L. Betancourt. 2003. Reevaluation of mid-Holocene deposits at Quebrada Piripica, northern Chile. Palaeogeography, Palaeoclimatology, Palaeoecology 194: 207-222.

Reese, C., K. Liu. 2005. A modern pollen rain study from the central Andes region of South America. Journal of Biogeography 32: 709-718.

Restifo, F. 2017. Assemblages, artifact and attributes as three levels of analysis relevant to the understanding of lithic raw material selection: a case study from the South Central Andes (Pastos Grandes Bassin, Salta Province, Argentina). En: $11^{\text {th }}$ International Symposium on Knappable Materials. "From Toolstone to Stone Tools". IMHICIHU CONICET, Buenos Aires: 56.

Restifo, F., Carbonelli, J. P. 2016. Una aproximación inicial a la variabilidad de puntas de proyectil de Puna y valles del Noroeste argentino. En: Actas del XIX Congreso Nacional de Arqueología Argentina. Serie Monográfica y Didáctica Volumen 54, Facultad de Ciencias Naturales e I.M.L., Universidad Nacional de Tucumán, Tucumán: 1292-1296.
Schabitz, F. 2000. Vegation and climate history on the eastern flank of the Santa Victoria, Jujuy Province, NW Argentina (first results). Zent. bl. Geol. Palaontol. 1 7/8: $1-16$.

Schabitz, F., L. Lupo, J. Kulemeyer, J. Kulemeyer. 2001. Variaciones en la vegetación, el clima y la presencia humana, durante los últimos 15000 años, en el borde oriental de la Puna, Provincia de Jujuy y Salta, noroeste argentino. Ameghiniana. Publicación Especial 8: 125-130.

Schittek, K. 2014. "Cushion peatlands in the high Andes of northwestern Argentina as archives for palaeoenvironmental Research". Dissertation Botanicae 412.

Stuiver, M. y P. Reimer. 1993. Extended 14C database and revised CALIB radiocarbon calibration program. Radiocarbon 35: 215-230.

Sylvestre, F., M. Servant, S. Servant-Vildray, C. Causse, M. Fournier y J. P. Ybert. 1999. Lake-level chronology on the southern Bolivian Altiplano (18 $\left.-23^{\circ} \mathrm{S}\right)$ during late-glacial time and the early Holocene. Quaternary Research 51: 54-66.

Tchilinguirian, P., M. Morales. 2013. Mid-Holocene Paleoenvironments in Northwestern Argentina: Main Patterns and Discrepancies. Quaternary International 307: 14-23.

Tchilinguirian, P., M. R. Morales, B. Oxman, L. C. Lupo, D. E. Olivera y H. D. Yacobaccio. 2012. Early to Middle Holocene transition in the Pastos Chicos record, dry Puna of Argentina. Quaternary International 330: 171-182.

Tchilinguirian, P., M. Morales, B. Oxman, M. Pirola. 2014. Paleoenvironmental studies of the Quebrada de Lapao, Jujuy Province, Argentina ( $23^{\circ} 22^{\prime} 01^{\prime \prime} \mathrm{S}, 66^{\circ} 21^{\prime} 52,8^{\prime \prime}$ W, 3650 M A.S.L.) for the $9400-7300$ Yrs B.P. span. En D. M. Kligmann y M. R. Morales (eds.), Physical, Chemical and Biological Markers in Argentine Archaeology: Theory, Methods and Applications: 87-104. Oxford, BAR International Series 2678.

Thompson, L., E. Mosley- Thompson, M. Davis, P. -N. Lin, K. Henderson, J. Cole-Dai, K. Liu. 1995. Late glacial stage and Holocene tropical ice core records from Huscarán, Peru. Science 269: 46-50.

Thompson, L., Davis, M., Mosley Thompson, E., Sowers, T.A., Henderson, K., Zagorodnov, V.S., Lin, P.N., Mikhalenko, V.N., Campen,r.k., Bolzan, J.F., Cole-Dai, J., B. Francou. 1998. A 25000 year tropical climate history from bolivian ice cores. Science 282:1858-1864.

Yacobaccio, H. D. 1994. Biomasa animal y consumo en el Pleistoceno-Holoceno Surandino. Arqueología 4: 43-71. 
Yacobaccio, H. D.2013. Towards a Human Ecology for the Middle Holocene in the Southern Puna. Quaternary International 307: 24-30.

Yacobaccio, H. D., Morales, M. R. 2013. Ambientes pleistocénicos y ocupación humana temprana en la Puna argentina. Boletín de Arqueología PUCP N¹5: 337-356.

Yacobaccio, H. D., Vilá, B. 2002. Condiciones, Mecanismos y Consecuencias de la Domesticación de los Camélidos. Estudios Sociales del NOA, Año 5, N5: 4-27.

Yacobaccio, H. D., Catá, M. P., Solá, P., Alonso, M. S. 2008. Estudio arqueológico y físico-químico de pinturas rupestres en Hornillos 2 (Puna de Jujuy). Estudios Atacameños 36: 5-28.

Yacobaccio, H. D., Hoguin, R., Glascock, M. 2017. Obsidian supply and circulation in Puna of Jujuy province.
En: $11^{\text {th }}$ International Symposium on Knappable Materials. "From Toolstone to Stone Tools". IMHICIHU CONICET, Buenos Aires: 93.

Yacobaccio, H. D., Madero, C. M., Malmierca, M. P., Reigadas, M. C. 1997/1998. Caza, domesticación y pastoreo de camélidos en la Puna Argentina. Relaciones Tomo XXII-XXIII: 389-418.

Yacobaccio, H. D., Morales, M. R., Solá, P., Samec, C. T., Hoguin, R., Oxman, B. 2013. Mid-Holocene Occupation in the Dry Puna in NW Argentina: Evidence from the Hornillos 2 rockshelter. Quaternary International 307: 38-49.

Yacobaccio, H. D., Escola, P., Pereyra, F., Lazzari, M., Glascock, M. 2004. Quest for ancient routes. Obsidian sourcing research in Northwestern Argentina. Journal of Archaeolocical Science 31: 193-204. 